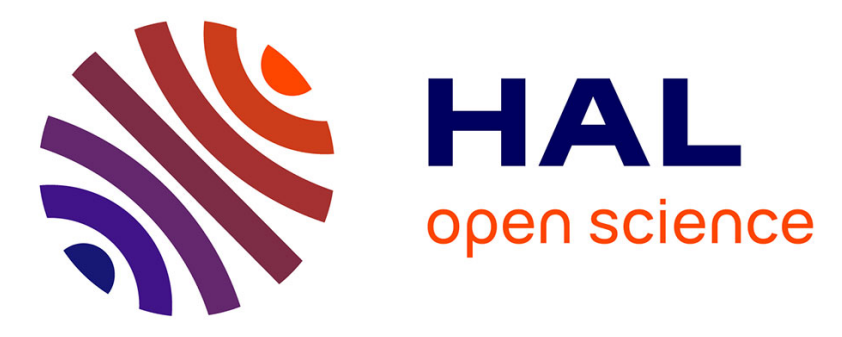

\title{
Sun backward gazing method for measuring optomechanical errors of solar concentrators: experimental results
}

F. Henault, Cyril Caliot, M. Coquand, P.-H. Defieux, Emmanuel Guillot

\section{- To cite this version:}

F. Henault, Cyril Caliot, M. Coquand, P.-H. Defieux, Emmanuel Guillot. Sun backward gazing method for measuring optomechanical errors of solar concentrators: experimental results. Applied optics, 2020, 59 (31), pp.9861. 10.1364/AO.399595 . hal-03006761

HAL Id: hal-03006761

https: / hal-univ-pau.archives-ouvertes.fr/hal-03006761

Submitted on 16 Nov 2020

HAL is a multi-disciplinary open access archive for the deposit and dissemination of scientific research documents, whether they are published or not. The documents may come from teaching and research institutions in France or abroad, or from public or private research centers.
L'archive ouverte pluridisciplinaire HAL, est destinée au dépôt et à la diffusion de documents scientifiques de niveau recherche, publiés ou non, émanant des établissements d'enseignement et de recherche français ou étrangers, des laboratoires publics ou privés. 


\title{
Sun backward gazing method for measuring opto-mechanical errors of solar concentrators. First experimental results
}

\author{
F. Henault ${ }^{1, *}$, C. Caliot ${ }^{2,3}$, M. Coquand ${ }^{2}$, P.-H. Defieux ${ }^{2}$ and E. \\ GUILLOT $^{2}$ \\ ${ }^{1}$ Institut de Planétologie et d'Astrophysique de Grenoble, IPAG CNRS, Université Grenoble Alpes F- \\ 38041 Grenoble, France \\ ${ }^{2}$ Processes, Materials and Solar Energy laboratory, PROMES CNRS, 7 Rue du Four Solaire, 66120 \\ Font-Romeu-Odeillo-Via, France \\ ${ }^{3}$ Laboratoire de Mathématiques et de leurs Applications, Université de Pau et des Pays de l'Adour, E2S \\ UPPA, CNRS, LMAP, Allée du parc Montaury, 64600 Anglet, France \\ *francois.henault@univ-grenoble-alpes.fr
}

\begin{abstract}
This paper presents the first experimental results obtained with the four cameras backward gazing method for measuring the opto-mechanical errors of solar concentrating surfaces in operation. It consists in combining the images simultaneously acquired by four cameras placed near the solar receiver in order to reconstruct the slopes errors of the mirrors digitally. New algorithms adapted to real acquired images are described. The experiment has been conducted on a sun-tracking heliostat of the experimental solar tower plant THEMIS with slopes error measurement accuracy estimated as $0.2 \mathrm{mrad}$. The capacity of characterizing shape distortions due to wind or gravity loads is also investigated. The main experimental error sources are analysed and possible ways of mitigation are proposed, giving reasonable hope in reaching an ultimate measurement accuracy of $0.1 \mathrm{mrad}$.
\end{abstract}

(C) 2020 Optical Society of America under the terms of the OSA Open Access Publishing Agreement

\section{Introduction}

Concentrated solar power (CSP) is a promising method for renewable energy production in the future. Whatsoever the type of the solar power plant, e.g. solar tower plant, parabolic dish or trough, the pointing and canting accuracies of the solar concentrating surfaces have a huge impact on the solar flux distribution on the receiver, which can affect the efficiency of the power plant. It is then necessary to develop mirror alignment and control methods operable in a reasonable period of time at the industrial scale. In addition, it is desirable that the mirrors are measured in operation (i.e. in sun-tracking mode) in order to evaluate environmental effects such as ambient temperature, wind, gravity load variation for different mirror orientations, or ageing. Finally, the measurements should be carried out without disturbing the heat production process. With the ongoing development of CSP, many methods for mirror alignment and control were proposed and tested, such as summarized in the review articles [1-3]. It follows that nowadays the two most popular methods seem to be photogrammetry and deflectometry. The former was tested successfully several times [4], but is timeconsuming and requires the mirror to be in a static position since specific test equipments must be mounted to it (measurement targets and cameras). Therefore it is not applicable to a sun-tracking mirror. On the other hand, deflectometry methods consist in observing the image of a pre-determined object seen through the tested optical surface, and to evaluate its surface errors from the distorted image of the object. Moss and Diver have for example applied this method to parabolic trough systems [5], but it requires setting the mirrors in fixed vertical position. For solar tower plants, Ulmer et al [6] describe another deflectometry method that consists in observing the deformation of a stripe pattern located below the solar receiver, from a camera located above it. Here again the heliostat must be static, and oriented so as to reflect 
the image of the stripes towards the camera. The measurement accuracy of this method can be improved by introducing temporal modulation of the stripe pattern [7].

So far it seems that the sole measurement principle enabling in situ characterization of solar concentrating surfaces is the backward gazing method that was initially developed in the eighties to evaluate the canting errors of the French 1-MW solar furnace in Odeillo [8]. The modern version of this method makes use of four cameras located at the vicinity of the solar receiver, and simultaneously acquiring the images of a heliostat in sun-tracking mode. The images are then processed digitally in order to reconstruct the slopes errors of the observed mirror. Numerical simulations of the achievable measurement accuracy and improvements of the slopes reconstruction algorithms have been the scope of recent publications [9-12]. It has also been shown that the method can be generalized to the case of a grid of $N$ x $N$ observing cameras with $N \geq 2$, enabling improved measurement accuracy and extended mirror surface coverage [13]. In the present article are presented the first experimental results of the backward gazing method with four cameras. The basic principle of the method is firstly summarized in section 2, including the last updates in terms of digital processing. The experimental configuration and procedure are described in section 3. The main experimental results are given in section 4 and are discussed in light of the major identified error sources and of their possible mitigation. A brief conclusion on the potential of the method is finally given in section 5 .

\section{Backward gazing method with four cameras. Principle and updates}

The general principle of the backward gazing method with four cameras is firstly summarized in section 0 . Then the major updates that essentially concern the data processing software are described in section 0 .

\subsection{General principle}

The basic principles of the backward gazing method are extensively described in Refs. [9-10]. It essentially makes use of four cameras located at the vicinity of the solar receiver and simultaneously recording images of the Sun reflected by the optical surface of an heliostat. Depending on the location of the cameras, the intensity distributions observed through the mirror do not exactly match, and their relative differences are used to retrieve the surface slopes errors digitally at any point $\mathrm{P}$ of the mirror (see Figure 1). The optical errors of the heliostat are then reconstructed from the measured slopes. Data processing allows identifying various types of errors, i.e. tracking error of the heliostat, canting errors of its individual facers, and shape errors affecting the facets. The employed notations and coordinate systems are illustrated in Figure 2 and Figure 3: The main reference frames are defined as:

- The reference frame of the solar facility $\mathrm{R}_{\mathrm{F}}\left(\mathrm{FX}_{\mathrm{F}} \mathrm{Y}_{\mathrm{F}} \mathrm{Z}_{\mathrm{F}}\right)$, where $\mathrm{F}$ is a common reference point usually located at the bottom of the solar tower (not shown in Figure 2), $\mathrm{FX}_{\mathrm{F}}$ and $\mathrm{FY}_{\mathrm{F}}$ axes are horizontal axes are directed southward and eastward respectively, and the $\mathrm{FZ}_{\mathrm{F}}$-axis is directed to Zenith. The azimuth and elevation angles of the Sun vector $\mathbf{S}_{\mathbf{0}}$ directed at the Sun centre are noted $a_{\mathrm{S}}$ and $h_{\mathrm{S}}$ in that coordinate system (bold characters denoting vectors in the whole text and figures).

- The heliostat reference frame $\mathrm{R}_{\mathrm{H}}(\mathrm{OXYZ}$ ), where $\mathrm{O}$ is the heliostat centre, the OYZ plane is tangent to the reflecting surface at point $\mathrm{O}$, and $\mathrm{OX}$ axis is parallel to the pointing vector $\mathbf{N}_{\mathbf{0}}$ of the heliostat. Any point $\mathrm{P}$ of the optical surface is defined by its three Cartesian coordinates $(x, y, z)$. Because the heliostat is not generally flat, the $x$ coordinate of point $\mathrm{P}$ depends on its $y$ and $z$ coordinates and can be written as:

$x(y, z) \approx\left(y^{2}+z^{2}\right) / 4 D+\Delta(P)$, 
where $D$ is the distance $\left\|\mathrm{OO}^{\prime}\right\|$ from the heliostat to the target point (see Figure 3 ) and $\Delta(\mathrm{P})$ are the shape errors to be measured, including tracking, canting, and local surface errors of the different modules. The first quadratic term in Eq. 1 indicates that these errors are measured with respected to a spherical reference surface (limited to its second-order terms) centred at $\mathrm{O}$ and of focal length equal to $D$.

- The target vector reference frame $\mathrm{R}^{\prime}\left(\mathrm{O}^{\prime} \mathrm{X}^{\prime} \mathrm{Y}^{\prime} \mathrm{Z}^{\prime}\right)$, with $\mathrm{O}^{\prime}$ the target point usually located at or near to the solar receiver, and the $\mathrm{O}^{\prime} \mathrm{X}^{\prime}$ axis is parallel to the target vector $\mathbf{R}_{\mathbf{0}}=\mathrm{OO}^{\prime} /\left\|\mathrm{OO}^{\prime}\right\|$ of the heliostat. The O'Y' axis is taken to be horizontal and $\mathrm{O}^{\prime} \mathrm{Z}$ ' completes the reference trihedra. Obviously, vectors $\mathbf{S}_{\mathbf{0}}, \mathbf{N}_{\mathbf{0}}$ and $\mathbf{R}_{\mathbf{0}}$ are linked together by the Snell-Descartes law of reflection, here expressed in a vector form:

$\mathbf{S}_{\mathbf{0}}+\mathbf{R}_{\mathbf{0}}=2\left(\mathbf{S}_{\mathbf{0}} \mathbf{N}_{\mathbf{0}}\right) \mathbf{N}_{\mathbf{0}}$.

- The four cameras are actually located in a target plane that is generally not strictly perpendicular to vector $\mathbf{R}_{\mathbf{0}}$, thus another reference frame R" (O'X'Y"Z") is attached to it, where the O'X" axis is perpendicular to the target plane. Any point $\mathrm{M}^{\prime}$ " in the O'Y"Z" plane is defined by its Cartesian coordinates $(0, y ", z$ "). Assuming the cameras to be located at the four corners $\mathrm{M}^{\prime \prime}{ }_{i j}$ of a rectangle of widths $2 \delta y$ " and $2 \delta z$ " and centred at point O", their coordinates are written as $\left(0, y^{\prime \prime}{ }_{i j}, z^{\prime \prime}{ }_{i j}\right)=(0, \pm \delta y ", \pm \delta z)$, with indices $i$ and $j$ varying between 1 and 2 .

- A Sun reference frame $\mathrm{R}_{\mathrm{S}}(\mathrm{OUVW})$ is finally used, with $\mathrm{U}$-axis parallel to the Sun vector $\mathbf{S}_{\mathbf{0}}$. Other points of the solar disk are localized by means of the angles $(v, w)$ respectively standing for rotations around the $\mathrm{W}$ and $\mathrm{V}$ axes.

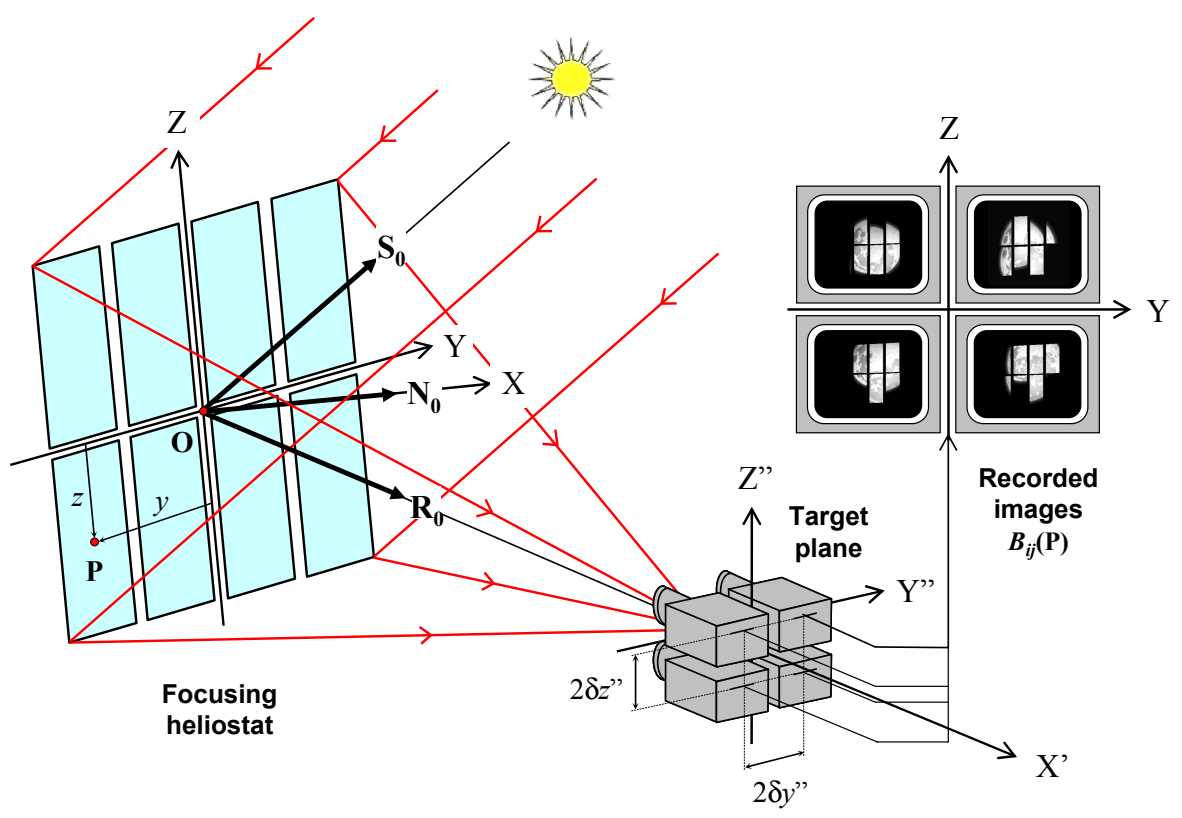

Figure 1.Principle of the four cameras backward gazing method. 
The backward gazing method also requires a priori knowledge of the Sun's radiance profile, or sunshape function noted $B(\varepsilon)=\mathrm{B}_{0} f(\varepsilon)$, where $\mathrm{B}_{0}$ is the brightness at Sun centre and $f(\varepsilon)$ is a monotonously decreasing function from $f(0)=1$ at the Sun centre to $f\left(\varepsilon_{0}\right)=0$ at the rim of the solar disk: here $\varepsilon_{0}$ is the angular radius of the Sun $\varepsilon_{0}$, usually taken equal to 32 arcmin. Since $f(\varepsilon)$ is invertible on the interval $\left[0, \varepsilon_{0}\right]$, we define the $\mathrm{K}_{i j}(\mathrm{P})$ function as the square of the inverse function $f^{-1}(\varepsilon)$, i.e.:

$$
\mathrm{K}_{i j}(\mathrm{P})=\left[f^{-1}\left(\frac{\mathrm{B}_{i j}(\mathrm{P})}{\mathrm{B}_{0}}\right)\right]^{2},
$$

where $\mathrm{B}_{i j}(\mathrm{P})$ are the four images simultaneously acquired by the different cameras (with indices $1 \leq i \leq 2$ and $1 \leq j \leq 2$ ). In the framework of first-order geometrical optics [9], it has been shown that functions $\mathrm{K}_{i j}(\mathrm{P})$ give direct access to the partial derivatives $\partial W(\mathrm{P}) / \partial y$ and $\partial W(\mathrm{P}) / \partial z$ of the Wavefront error (WFE) $W(\mathrm{P})$ reflected by the heliostat:

$$
\left(\begin{array}{l}
\frac{\partial W(P)}{\partial y} \\
\frac{\partial W(P)}{\partial z}
\end{array}\right)=D\left(\begin{array}{l}
\frac{\mathrm{K}_{22}(\mathrm{P})+\mathrm{K}_{21}(\mathrm{P})-\mathrm{K}_{12}(\mathrm{P})-\mathrm{K}_{11}(\mathrm{P})}{8 \delta y^{\prime \prime}} \\
\frac{\mathrm{K}_{22}(\mathrm{P})-\mathrm{K}_{21}(\mathrm{P})+\mathrm{K}_{12}(\mathrm{P})-\mathrm{K}_{11}(\mathrm{P})}{8 \delta z^{\prime \prime}}
\end{array}\right),
$$

which can be interpreted as generalized quad-cell formulas. Deriving the shape error function $\Delta(\mathrm{P})$ of the heliostat from the slopes of the wavefront error function $W(\mathrm{P})$ is achieved using the following matrix transformation, also in first-order approximation:

$$
\left(\begin{array}{c}
\frac{\partial \Delta(P)}{\partial y} \\
\frac{\partial \Delta(P)}{\partial z}
\end{array}\right)=\frac{1}{2}\left[\begin{array}{cc}
\frac{1}{\cos H} & -\tan A \tan H \\
0 & \frac{1}{\cos A}
\end{array}\right]\left(\begin{array}{c}
\frac{\partial W(P)}{\partial y}-\frac{\partial W_{R}(P)}{\partial y} \\
\frac{\partial W(P)}{\partial z}-\frac{\partial W_{R}(P)}{\partial z}
\end{array}\right),
$$

where $A$ and $H$ are the azimuth and elevation angles of the Sun vector $\mathbf{S}_{\mathbf{0}}$ expressed in the heliostat coordinates frame $\mathrm{R}_{\mathrm{H}}$ as illustrated in Figure 3 , and $\partial W_{R}(\mathrm{P}) / \partial y$ and $\partial W_{R}(\mathrm{P}) / \partial z$ are the optical aberrations of the spherical reference surface defined by Eq. 1, to be subtracted to the measured wavefront slopes. Those terms essentially stand for astigmatism and curvature field aberrations. They can either be calculated by using their first-order developments as function of the $(y, z)$ coordinates (see Ref. [10], Eqs. 8), or with the help of a ray-tracing software which allows correcting higher order terms than the first one.

The last digital processing steps consist in reconstructing the shape error function $\Delta(\mathrm{P})$ from its estimated partial derivatives $\partial \Delta(\mathrm{P}) / \partial y$ and $\partial \Delta(\mathrm{P}) / \partial z$, then identifying the main types of errors affecting the heliostat. It should be noted that some of these errors can be characterized directly from the surface slopes without need for reconstructing the function $\Delta(\mathrm{P})$ itself: for example, canting errors appear as slopes offsets averaged over the surface of an individual facet, and tracking error as the global slopes offset over the whole surface of the heliostat (see Ref. [12]). 


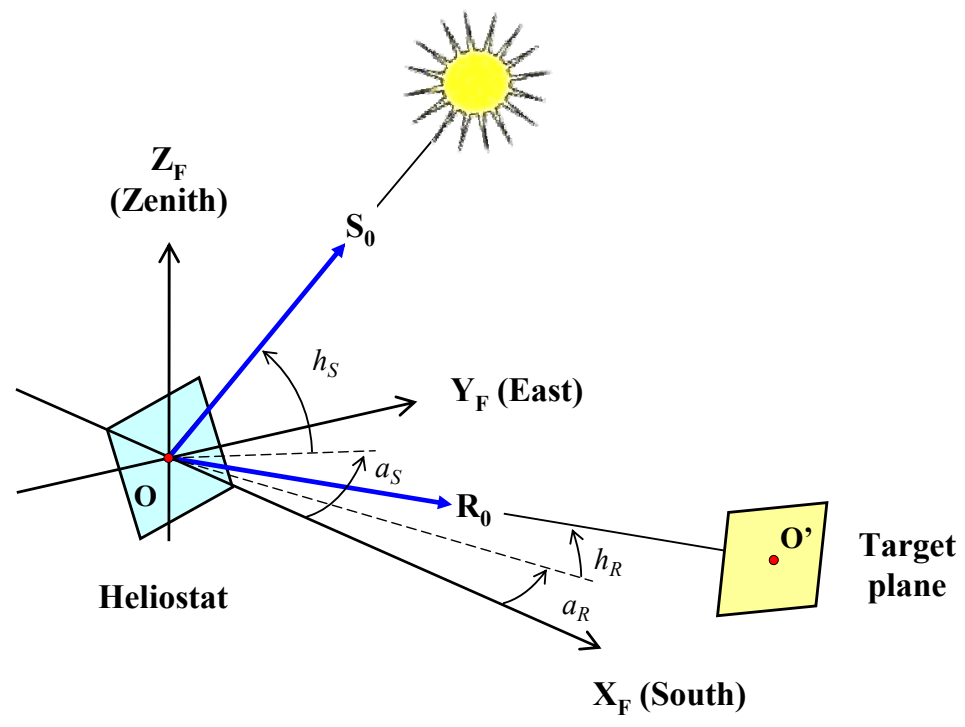

Figure 2: Definition of the solar facility reference frame $R_{F}$.

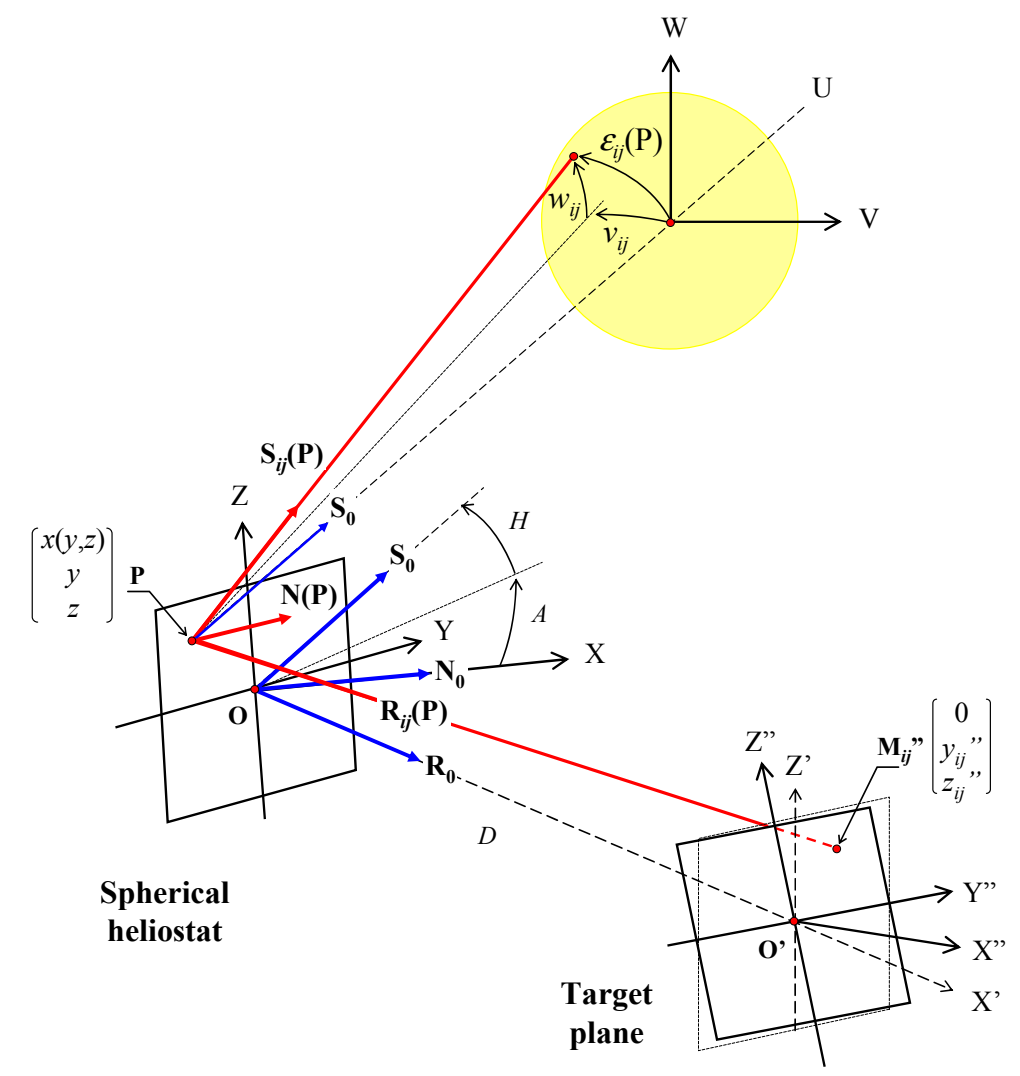

Figure 3: Main employed notations and coordinate systems. 
From Eqs. 3-5 a basic shape errors reconstruction procedure has been defined and tested by means of extensive numerical simulations, demonstrating that slopes measurement accuracy well below $0.1 \mathrm{mrad}$ are achievable in presence of various types of noise [9-12]. For digital processing of experimental images, which is the main scope of this paper, it is however mandatory to use enhanced algorithms for two main reasons:

- Eqs. 4-5 are only valid in the frame of first-order optics, which may prove insufficient if the measurements are carried out on heliostats reflecting solar rays under large incidence angles (typically $>20$ degrees). Moreover, Eq. 5 implicitly assumes that the target vector $\mathbf{R}_{\mathbf{0}}$ is parallel to the $\mathrm{OX}_{\mathrm{F}}$-axis, i.e. horizontal southward, and that the target plane is perpendicular to it, which is not generally the case.

- Also, these relations do not represent the best solutions for $\partial \Delta(\mathrm{P}) / \partial y$ and $\partial \Delta(\mathrm{P}) / \partial z$ in presence of experimental noises. Thus in a first step Eqs. 4 have been replaced with a digital optimization procedure that allows finding the best compromise between the four measured intensities $\mathrm{B}_{i j}(\mathrm{P})$, for each observed point $\mathrm{P}$ at the surface of the mirror [11-12]. Recently, the same logic has been applied to Eqs. 5, now being replaced by a reverse ray-tracing optimization procedure [14].

Finally, additional processing algorithms are required when dealing with real images, such as radiometric calibration, geometrical transformations or images registration. This led us to develop new or improved algorithms applicable to the measured images, which are described in the next sub-section.

\subsection{Processing algorithms}

A flow-chart of the main employed numerical procedures and algorithms is depicted in Figure 4. They start with the pre-processing of direct Sun images (1) and heliostat images $\mathrm{B}_{i j}(\mathrm{P})(2)$, then proceed with geometrical transformations of the images of the heliostat such as derotation and rectification (3). The two following steps (4-5) are critical for correct mirror slopes reconstruction: they consist in accurate registration of the four acquired images of the heliostat, so that their centres and edges coincide at sub-pixel level. The images are then prepared to WFE and mirror slopes reconstruction (6). Optionally, first-guess estimates of the mirror slopes $\partial \Delta(\mathrm{P}) / \partial y$ and $\partial \Delta(\mathrm{P}) / \partial z$ can be calculated from the theoretical model described in the previous sub-section (7). The last step consists in reconstructing the slopes errors of the heliostat by using the reverse ray-tracing optimization procedure (8). For each of these steps, details about the employed procedures or algorithms are provided below. 


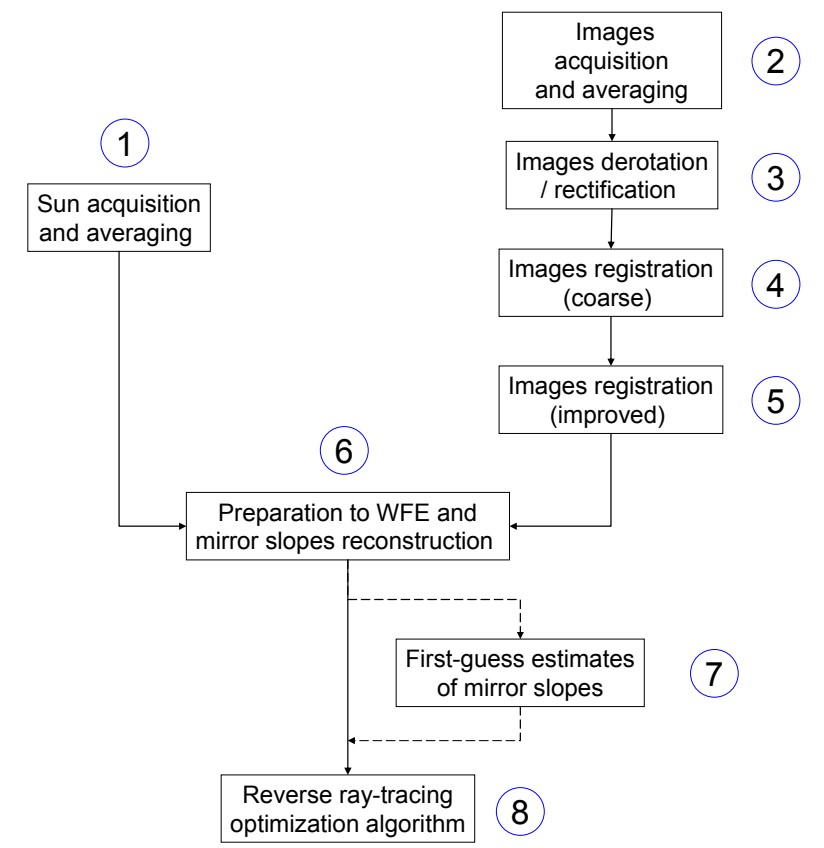

Figure 4: Flow-chart of the main employed procedures and algorithms.

\section{1) Sun acquisition and averaging}

$\mathrm{N}$ direct, reference images of the $\operatorname{Sun} B_{0}^{n}(v, w)$ are acquired, where $(v, w)$ are the angular coordinates inside the solar disk (see Figure 3 ) and $1 \leq n \leq \mathrm{N}$. Each of these images are firstly corrected for camera optics and detector non-homogeneities (flat-field correction), which were previously calibrated in the optical shop by use of an integrating sphere [12]. The median of the N images $\bar{B}_{0}(v, w)$ is then computed: it is constituted of the median value of each pixel. A single image per camera is obtained based on the median value of each pixel considering $\mathrm{N}$ values obtained from $\mathrm{N}$ images with the same camera. The median image is further expressed in polar coordinates as $\bar{B}_{0}(\varepsilon, \varphi)$ with $\varepsilon=\sqrt{v^{2}+w^{2}}$ and $\varphi=\arctan (w / v) .200$ radial profiles $\bar{B}_{0}\left(\varepsilon, \varphi_{k}\right)$ are interpolated for equally-spaced polar angles $\varphi_{k}$ and averaged together as $\overline{\bar{B}}_{0}(\varepsilon)=\left\langle\bar{B}_{0}\left(\varepsilon, \varphi_{k}\right)\right\rangle_{k}$ with $1 \leq k \leq 200$. The curve is then normalized as $f(\varepsilon)=\overline{\bar{B}}_{0}(\varepsilon) / \overline{\bar{B}}_{0}(0)$ and scaled along the horizontal axis so that its cut-off angle is matched to the nominal Sun radius, i.e. $f\left(\varepsilon_{0}\right)=0.5$. It may later be used in two different ways:

Either by simple linear interpolation of the sampling points; or:

By filling to the measured sunshape a "generalized Jose" formula inspired from Ref. [15], writing as:

$$
\begin{array}{ll}
B(\varepsilon)=B_{0} f(\varepsilon)=B_{0}\left(a+(1-a) \sqrt{1-\left(\varepsilon / \varepsilon_{0}^{\prime}\right)^{\xi}}\right) & \text { when } 0 \leq \varepsilon \leq \varepsilon_{0}^{\prime}, \text { and: } \\
B(\varepsilon)=0 & \text { otherwise, }
\end{array}
$$


where the parameters $a, \varepsilon_{0}^{\prime}$ and $\xi$ are optimized to match the measured $f(\varepsilon)$ curve. Examples of a median Sun map $\bar{B}_{0}(v, w)$ and its resulting averaged brightness profile are depicted in Figure 5.
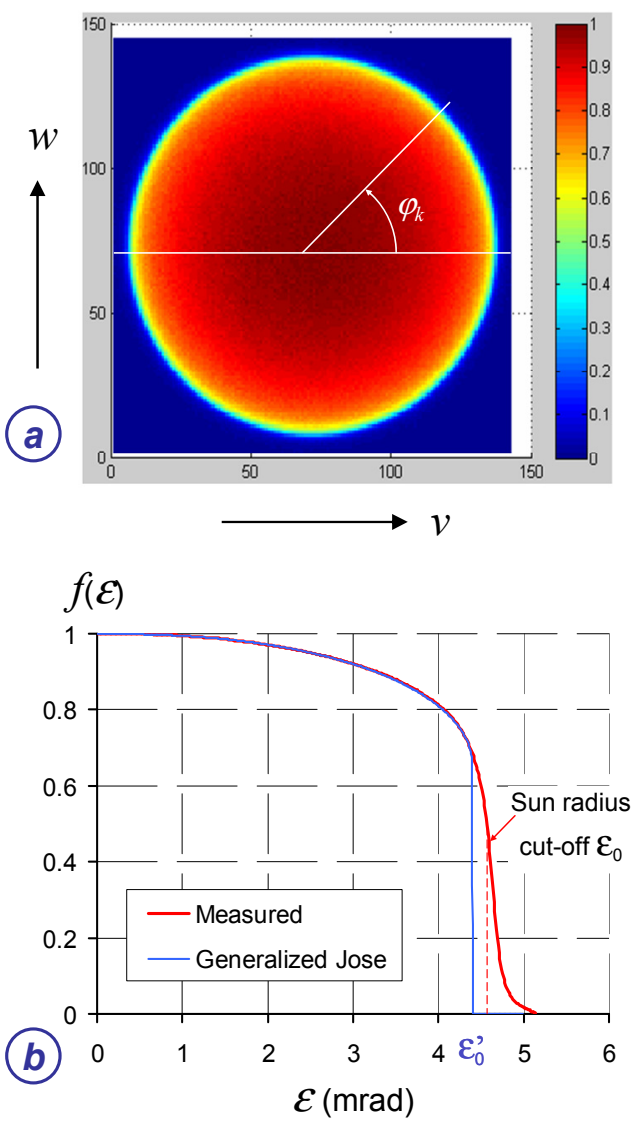

Figure 5: Median of 100 acquisitions of the Sun (a) and averaged brightness profile (b).

\section{2) Images acquisition and averaging}

For each of the four cameras located at points $\mathrm{M}^{\prime \prime}{ }_{i j}$, a set of $\mathrm{N}$ ' images $B_{i j}^{n^{\prime}}(P)$ of the heliostat in sun-tracking mode is acquired (with $1 \leq n^{\prime} \leq \mathrm{N}^{\prime}$ ). The same flat-field correction as in the previous sub-section is carried out for all of them. Median images $\bar{B}_{i j}(P)$ are then computed for the four cameras in the same way as for direct Sun images. The last step consists in an inter-camera radiometric calibration that can be written mathematically as $B_{i j}(P)=G_{i j} \bar{B}_{i j}(P) / B_{\text {Max }}$, where the $G_{i j}$ coefficients are the averaged gain factors of each camera measured from direct Sun image acquisitions, and $B_{M a x}$ is equal to the maximal intensity found on the set of four images. This allows to compensating for differential gains of the detectors and to normalize the observed brightness distributions so that they can be translated in terms of angular errors by later use of the $f(\varepsilon)$ function in steps 7-8. 


\section{3) Images derotation/rectification}

The images of a heliostat in sun-tracking mode will generally appear as parallelograms, eventually affected with a slight pyramidal effect for those located close to the solar receiver. Moreover, differential roll angles between the cameras will add unwanted image rotations. Before further processing the four calibrated images $\mathrm{B}_{i j}(\mathrm{P})$ must be geometrically transformed into their original rectangular form (rectification), and their $\mathrm{Y}$ and $\mathrm{Z}$ axes should be made parallel (derotation). Also, the dimensions of the heliostat should be made identical into the four images. The employed procedure for achieving these geometrical transformations is described in Appendix A. It is restricted to the case when the distorted rectangular contours of the heliostat can be approximated to a parallelogram.

\section{4) Images registration (coarse)}

This first, coarse image registration procedure consists in identifying the external contours of the heliostat in the four rectified images $\mathrm{B}_{i j}(\mathrm{P})$, both along the $\mathrm{Y}$ and $\mathrm{Z}$ axes. For that purpose, two mono-dimensional "contour functions" are estimated along each axis. The mirror edges are identified as the locations of the first and last maxima of the contour curves. The main data processing steps are described in Appendix B1, enabling a recentring (registration) accuracy that was estimated to 2-3 camera pixels.

\section{5) Images registration (improved)}

After coarse registration of the four images $B_{i j}(P)$, one of them is taken as reference and the three remaining images are recentred with respect to it. This is achieved by use of crosscorrelating the previous contour functions as described in Appendix B2. It allows exploiting all the information enclosed in the contour curves, including the edges of each individual module of the heliostat. Doing so, it was found that the achieved registration accuracy is below one camera pixel, which was our initial goal.

\section{6) Preparation to WFE and mirror slopes reconstruction}

This step essentially consists in rescaling the four registrated images $B_{i j}(P)$ to the actual dimensions of the heliostat along the $\mathrm{Y}$ and $\mathrm{Z}$ axes. This is achieved by matching the contour locations evaluated from the contour functions computed in the previous registration steps to their theoretical locations $\left(-y_{\mathrm{Max}},+y_{\mathrm{Max}}\right)$ and $\left(-z_{\mathrm{Max}},+z_{\mathrm{Max}}\right)$; where $y_{\mathrm{Max}}$ and $z_{\mathrm{Max}}$ are the halfwidths of the heliostat along the $\mathrm{Y}$ and $\mathrm{Z}$ axes respectively. A mask function $M(\mathrm{P})$ is also deduced from the theoretical dimensions of the heliostat and of its individual modules. This mask is useful for eliminating points located outside the reflective surfaces from the acquired images, and for reducing the measured areas at the surface of each mirror facet (typically by 5 $\%)$. Pictures of a heliostat and of the mask function $M(\mathrm{P})$ are presented in Figure 6, also showing the useful dimensions of the heliostat and of its individual modules. This mask shall be applied to all experimental results presented in section 4 .

Additionally, and if first-guess estimates of the mirror slopes are used as explained in the next step, it is necessary to compute the angular parameters $A$ and $H$ as defined in section 0 . This last calculation is summarized in the Appendix C. 

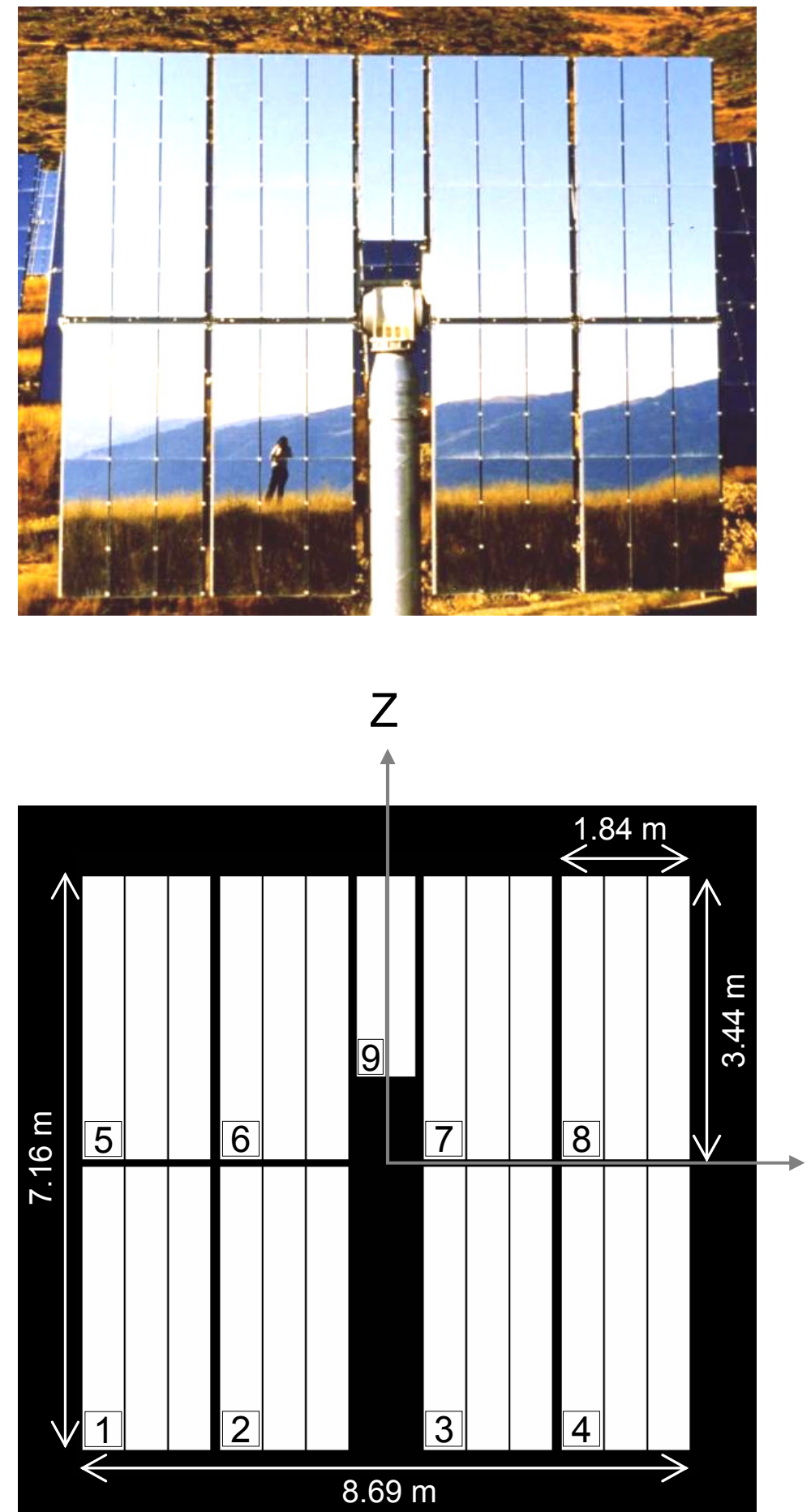

Figure 6: Picture of a CETHEL heliostat (top) and spatial dimensions of the applied mask function $M(\mathrm{P})$, showing the useful dimensions of the heliostat and the numbers and dimensions of its individual modules (bottom). 


\section{7) First-guess estimates of mirror slopes (optional)}

First-guess estimates of the searched mirror slopes $\partial \Delta(\mathrm{P}) / \partial y$ and $\partial \Delta(\mathrm{P}) / \partial z$ may be computed to feed the reverse ray-tracing optimization procedure described in the next and last step. The advantage of using first-guess solutions is twofold:

- They allow noticeable reduction of the required computing time, typically by a factor 3 or 4 , and:

- They drastically reduce the risk of finding secondary minimums of the cost function employed with the reverse ray-tracing optimization procedure described in the next section.

Practically, this step consists in applying the same error reconstruction procedure as described in Refs. [9-10]: using Eq. 3, the $\mathrm{K}_{i j}(\mathrm{P})$ functions are firstly determined from the registrated images $\mathrm{B}_{i j}(\mathrm{P})$ and knowledge of the normalized Sun radiance function $f(\varepsilon)$ measured at step 1 . The WFE slopes of the heliostat $\partial W(\mathrm{P}) / \partial y$ and $\partial W(\mathrm{P}) / \partial z$ are then evaluated from Eq. 4. The slopes of the heliostat shape errors $\partial \Delta(\mathrm{P}) / \partial y$ and $\partial \Delta(\mathrm{P}) / \partial z$ are finally deduced from the WFE slopes by using the matrix relationship 5, where the subtracted optical aberrations are approximated to their first-order development [10]:

$\frac{\partial W_{R}(P)}{\partial y}=\frac{x}{D} \cos A$ and: $\frac{\partial W_{R}(P)}{\partial z}=-\frac{x}{D} \sin A \sin H+\frac{y}{D} \cos H$.

\section{8) Reverse ray-tracing optimization algorithm}

This last part of the mirror slopes reconstruction procedure consists in launching rays from the four observing cameras at each point $\mathrm{P}$ located at the heliostat surface, where a priori unknown slopes errors $\partial \Delta(\mathrm{P}) / \partial y$ and $\partial \Delta(\mathrm{P}) / \partial z$ are introduced. After reflection at the mirror surface, these rays are propagated towards the solar disk and their angular coordinates $\left(v_{i j}, w_{i j}\right)$ are calculated in the Sun reference frame $\mathrm{R}_{\mathrm{S}}$. For each point $\mathrm{P}$ of the heliostat and cameras location $\mathrm{M}^{\prime \prime}{ }_{i j}$ the angular deviations of the rays with respect to the Sun centre $\varepsilon_{i j}(\mathrm{P})$ are computed (see Figure 3). The main steps of this reverse ray-tracing procedure are described in Appendix D. We then define a cost function $C F$ as:

$C F=\sqrt{\sum_{i=1}^{4}\left[R(P) f\left(\varepsilon_{i j}(P)\right)-B_{i j}(P)\right]^{2}}$,

where $\mathrm{R}(\mathrm{P})$ stands for a reflective factor at the heliostat surface typically close to unity, $f(\varepsilon)$ is the Sun normalized radiance function measured at step 1 , and $\mathrm{B}_{i j}(\mathrm{P})$ are the acquired and registrated images resulting from step 6 . The cost function is finally minimized with respect to the $\mathrm{R}(\mathrm{P}), \partial \Delta(\mathrm{P}) / \partial y$ and $\partial \Delta(\mathrm{P}) / \partial z$ parameters with the help of an optimization algorithm, here the AMOEBA procedure provided with the Interactive Data Language (IDL ${ }^{\mathrm{TM}}$ ) software package. It must be noted that this procedure enables full decoupling between the local reflection factor $\mathrm{R}(\mathrm{P})$ of the mirror and the measured slopes errors, thus eliminating the effect of soiling at the heliostat surface.

If available, the first-guess solutions found for $\partial \Delta(\mathrm{P}) / \partial y$ and $\partial \Delta(\mathrm{P}) / \partial z$ in step 7 are used to start the optimization process, otherwise their initial values are set to zero. Initial values for $\mathrm{R}(\mathrm{P})$ are always equal to unity. A quality criterion can also be set to the achieved values of the cost function $C F$ at the end of the optimization process: solutions found at any point $\mathrm{P}$ are eliminated if $C F$ is higher than a certain threshold value $t_{\mathrm{CF}}$, and the mask function $M(\mathrm{P})$ is then set to zero. 
Other employed algorithms are not described here, such as isolating the different modules of the heliostat, or estimating the average and standard deviations values of the mirror slopes over predefined surfaces. In the next section is described the experiment that was carried out to validate the principle of the method and of the data processing chain.

\section{Description of the experiment}

\subsection{THEMIS experimental solar tower facility}

The $105 \mathrm{~m}$ high THEMIS tower is part of a decommissioned solar tower power plant operated by Electricité de France (EDF) between 1983 and 1986 in Targasonne, France. Today, it is used by the CNRS-PROMES laboratory as an experimental research facility. The four cameras are located below the central receiver aperture at $76 \mathrm{~m}$ high (Figure 7) and at the back of the passively cooled white target plate protecting the acquisition devices (Figure 8). Each of them is placed behind a $25-\mathrm{mm}$ diameter opening pierced in the white target and enabling the observation of the heliostat field. The distance between these cameras was set to $\delta x^{\prime}=\delta y^{\prime}=200 \mathrm{~mm}$. A fifth camera located at the top of the tower is directed toward the Sun to measure its profile in real time (see Figure 7-b).
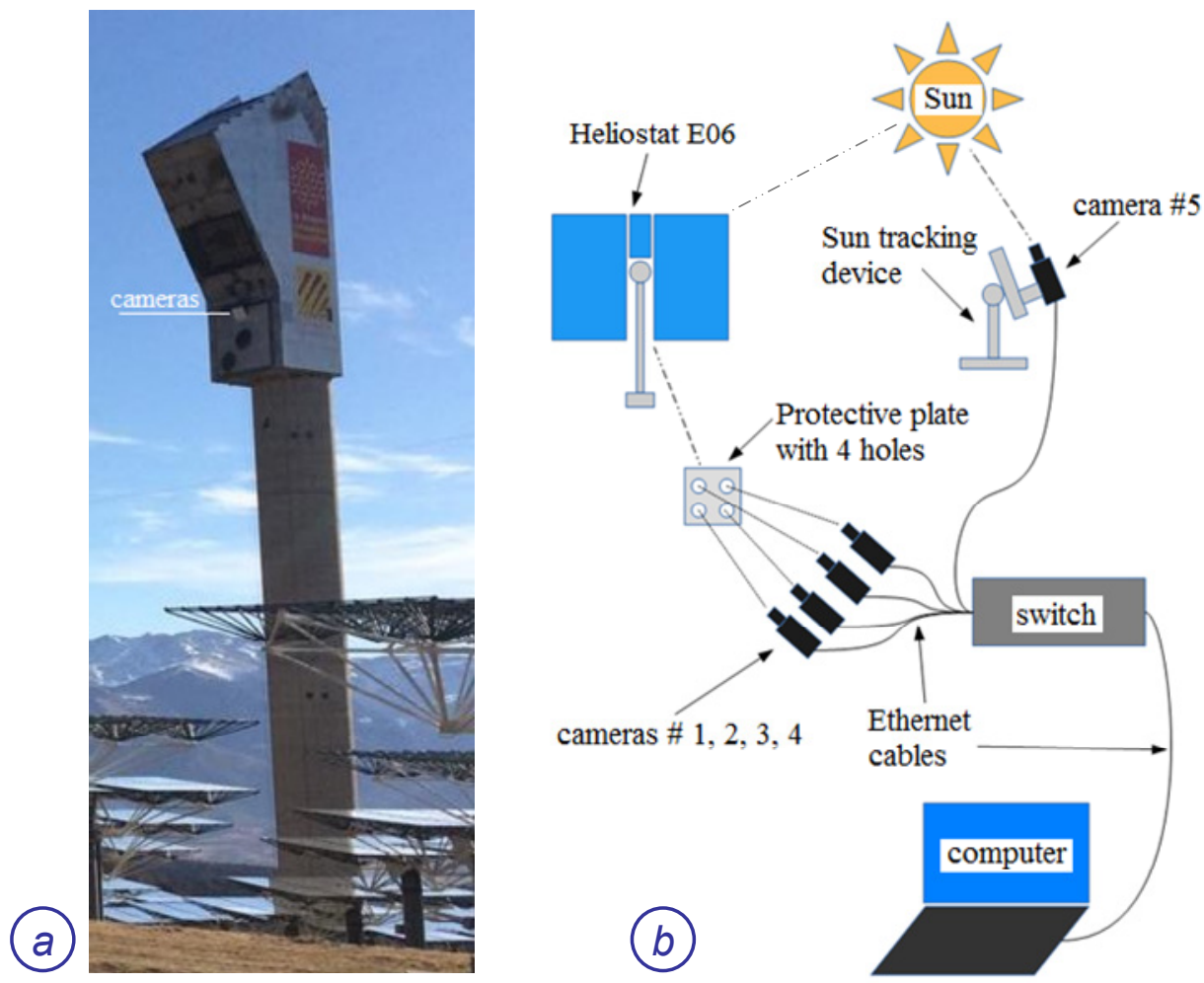

Figure 7: (a) THEMIS solar tower with the location of the cameras. (b) Schematic of the acquisition setup. 

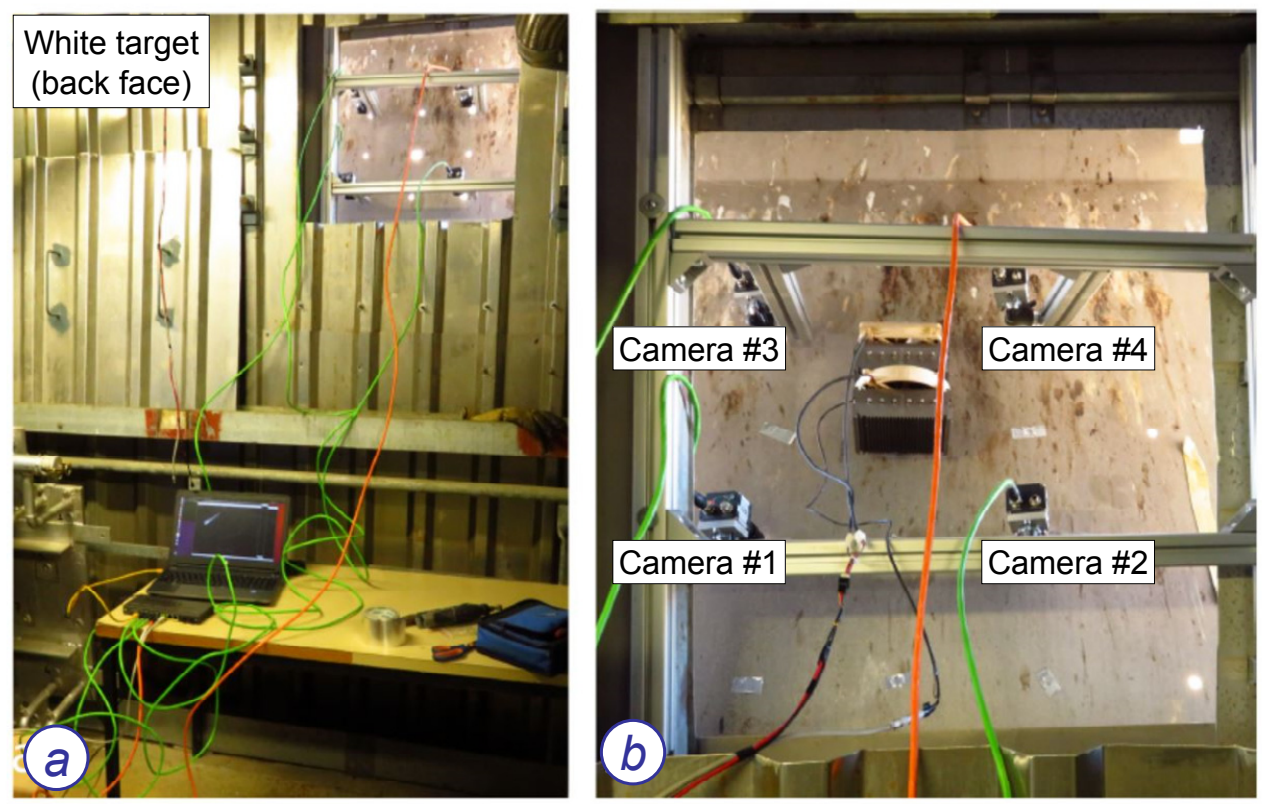

Figure 8: Pictures of the acquisition setup located at the back of the THEMIS white target plate. (a) General view. (b) View of the four cameras and their mechanical supports.

\subsection{THEMIS heliostats geometry}

The experiment was conducted for the heliostat numbered E06 which has the CETHEL III type and an area of $54 \mathrm{~m}^{2}(8.75 \mathrm{~m} \times 7.34 \mathrm{~m})$. Its focal length is $128 \mathrm{~m}$ and its centre $\mathrm{O}$ is located at a distance $\mathrm{D}=131.3 \mathrm{~m}$ from the target plane. The heliostat has nine modules (see Figure 6), from which eight are constituted of three mirror facets having a parabolic curvature along the $\mathrm{Z}$-axis and no curvature along the $\mathrm{Y}$-axis. The mirror facets of each module are tilted around the $\mathrm{Z}$ axis to approach a spherical surface with curvature radius $R_{\mathrm{m}}=2 f_{\mathrm{m}}$ (with $f_{\mathrm{m}}=140 \mathrm{~m}$ for heliostat E06). Then, each module is attached to a metallic structure with three set screws positioned at the vertices of a triangle, allowing independent adjustments in both azimuth (around Z-axis) and elevation (around Y-axis). A $9^{\text {th }}$ "complementary" module is located just above the elevation rotation mechanism, and is composed of two cylindroparabolic strips only.

\subsection{Hardware description}

Small cameras (UI-5240RE IDS) with a CMOS monochrome sensor are used to capture images of the Sun reflected through the heliostat at a maximal resolution of 1280x1024. An integrating sphere was used to calibrate the flat-field of each camera. To avoid sensor saturation and degradation during the measurements, two filters were mounted on a $1 \mathrm{~A} 1 \mathrm{HB}$ Tamron focusing lens with focal length $f=75 \mathrm{~mm}$. The first filter is essentially reflective, transmitting the beam flux with an optical density of $3(0.1 \%$ in the $400-700 \mathrm{~nm}$ range). The second presents an optical density of 7 which leads to a global attenuation of $100 \mathrm{~dB}$ for each camera. The responses of each lens and camera assembly are calibrated before experiment by recording simultaneously the intensity of the Sun (see $\S 0.2$ ).

The optical layout of the camera is illustrated in Figure 9. Point O' is actually located at the centre of the camera lens, whose useful diameter is equal to $d^{\prime}=25 \mathrm{~mm}$. Tested areas at the heliostat surface are optically conjugated with the plane of the detector array, which ensures negligible light contamination or crosstalk between different points $\mathrm{P}$ in the OYZ 
plane. The circular aperture of the camera lens also acts as a pinhole limiting diffuse cones of light reaching the detector to an angular radius $\delta \varepsilon=d^{\prime} / \mathrm{D}$ (solid and dashed red lines in the figure), thus filtering straylight from any point $\mathrm{P}$. For the E06 heliostat $\delta \varepsilon$ is around $0.1 \mathrm{mrad}$, which matches our initial measurement accuracy goal (see $\S 0$ ) and prevents the experiment from bias errors generated by diffuse reflections due to heliostat soiling.

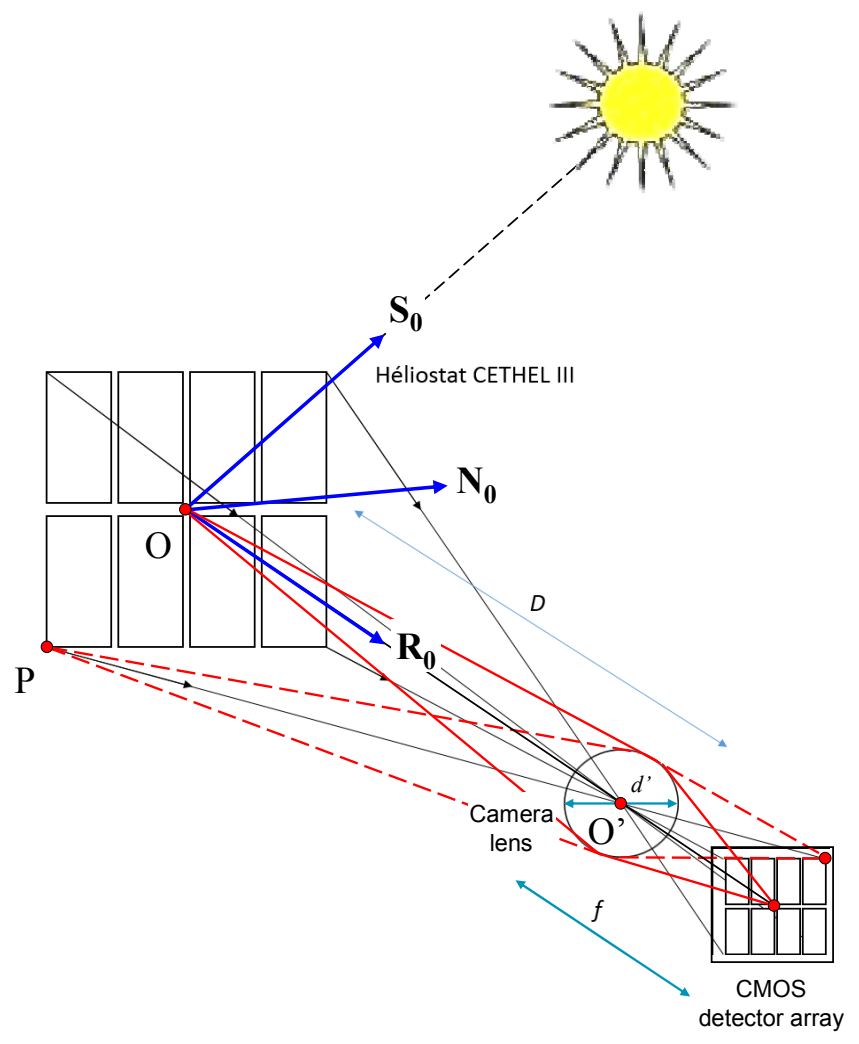

Figure 9: Camera layout illustrating the optical conjugation between heliostat and detector array (solid and dashed red lines).

The cameras are further attached to their mechanical supports located behind the white target plate (see Figure 8), where they are mounted on ball joints enabling coarse image centering and minimization of the roll angle $\theta_{Y i j}$ (see Appendix A). A switch with Ethernet cables (category 5) connects the cameras to the computer (Figure 7). Dedicated software using the OpenCV library was used to record the images.

\subsection{Measurement procedure}

The theoretical shape of the reflective surfaces of the CETHEL III heliostats is described analytically in Ref. [16], Appendix 3. However their actual shapes are poorly known. For example, the E06 heliostat has already been measured using photogrammetry or the optimization of focused Sun spots [17], but none of these methods provide measurement accuracy at the $0.1 \mathrm{mrad}$ level. To experimentally evaluate and validate the backward gazing method with four cameras, the experiment then consists in changing the orientation of two modules by turning their adjustment screws manually, and to compare the introduced defects with the reconstructed ones. This procedure is applied to the two lower modules located from each side of the heliostat pedestal (modules 2 and 3 in Figure 6). For both configurations 
(before and after defect introduction), a set of $\mathrm{N}^{\prime}=5$ images $B_{i j}^{n^{\prime}}(P)$ of the heliostat surface in sun-tracking mode is acquired for each of the four cameras with $1 \leq n^{\prime} \leq 5, i=1,2$ and $j=$ 1,2. Simultaneously, five direct Sun images $B_{0}^{n}(v, w)$ with $1 \leq n \leq 5$ are acquired by the fifth camera. The common acquisition time for all mages was set to 2 milliseconds. They are later processed digitally following the procedures described in section 0 .

\section{Experimental results}

After establishing a preliminary requirement for the mirror slopes measurement accuracy ( $\S$ $0)$, the main results obtained with the THEMIS experiment are presented both in terms of slopes errors $(\S 0)$ and reconstructed shape errors $(\S 0)$. Finally, $\S 0$ presents an analysis of the main identified experimental errors, and possible ways for mitigating them.

\subsection{Requirements}

The required alignment measurement accuracy for solar concentrating surface can be derived from their influence on the effective concentration ratio $\mathrm{C}$ of the optical surface. From the approximate relations in Ref. [18], the loss in concentration $\delta \mathrm{C}$ can be approximated as $\delta \mathrm{C} \approx$ $\left(\delta / \varepsilon_{0}\right)^{2}$, where $\delta$ is the standard deviation of the angular errors on the reflected rays, and $\varepsilon_{0}$ the angular radius of the Sun. $\delta$ is usually approximated as $2 \delta_{\mathrm{M}}$, with $\delta_{\mathrm{M}}$ the standard deviation of the mirror slopes errors. Assuming that $\delta \mathrm{C}$ must not exceed $4 \%$ leads to maximal angular errors $\delta_{\mathrm{M}}$ around $0.5 \mathrm{mrad}$. The desirable measurement accuracy is thus defined empirically as being five times less, i.e. $0.1 \mathrm{mrad}$. For differential measurements such as performed experimentally, it should translate into $\sqrt{2} \times 0.1 \approx 0.14 \mathrm{mrad}$.

\subsection{Slopes and canting errors}

This first set of measurement was carried out on the $21^{\text {st }}$ of December 2017 at $15 \mathrm{~h} 11$ local time, which correspond to solar angles $a_{\mathrm{S}}$ and $h_{\mathrm{S}}$ equal to $-34.3 \mathrm{deg}$. in azimuth and +19.9 deg. in elevation. Canting errors equal to $h_{2}=-1.33$ and $h_{3}=+0.89 \mathrm{mrad}$ in elevation were introduced respectively on modules 2 and 3 of the heliostat E06. These values correspond respectively to -1.5 and 1 turns applied manually to the lower screws of the modules. No canting errors were introduced in azimuth, i.e. $a_{2}=a_{3}=0 \mathrm{mrad}$. It has to be noted that the uncertainty on the introduced errors has been quantified as $0.27 \mathrm{mrad}$ [12], which is nearly twice our initial goal. The measurement accuracy of the pre-defined canting errors should then be limited by that last number.

The first step of the slopes reconstruction procedure consists in determining the Sun's normalized radiance profile $f(\varepsilon)$ as explained in $\S 0.1$. The generalized Jose formula in Eq. 6 was fitted to the actual sunshape acquired by the fifth camera with best-fit parameters $a=$ $4.406, \varepsilon_{0}^{\prime}=0.657 \mathrm{mrad}$ and $\xi=2.335$. The images of the heliostat are then geometrically transformed ( $\S 0.3)$ and registrated $(\S 0.4$ and 0.5$)$. The resulting rectified images seen from cameras 1 to 4 are depicted in Figure 10 and Figure 11, respectively for heliostat E06 in initial state and with canting errors added. 


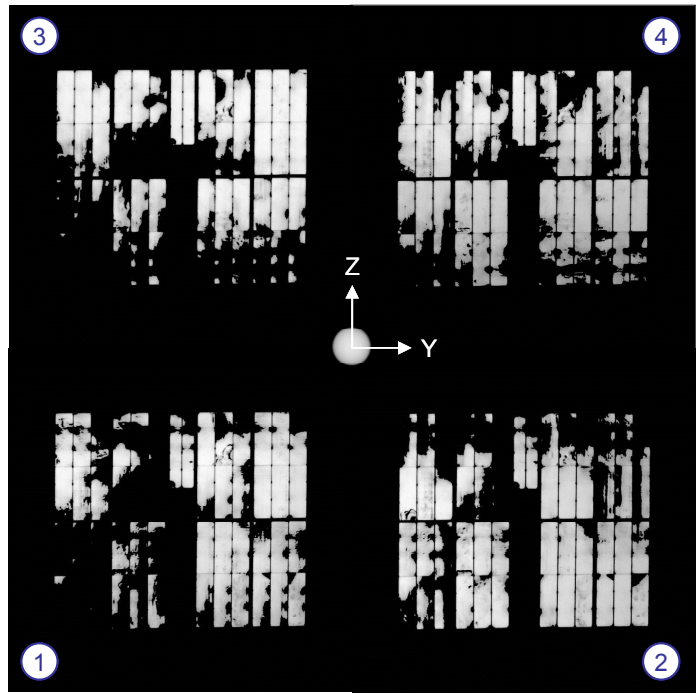

Figure 10: Rectified images of heliostat E06 in initial state, seen from cameras 1 to 4 . A direct Sun image captured by camera $\# 5$ is reproduced at the centre with same angular scale.

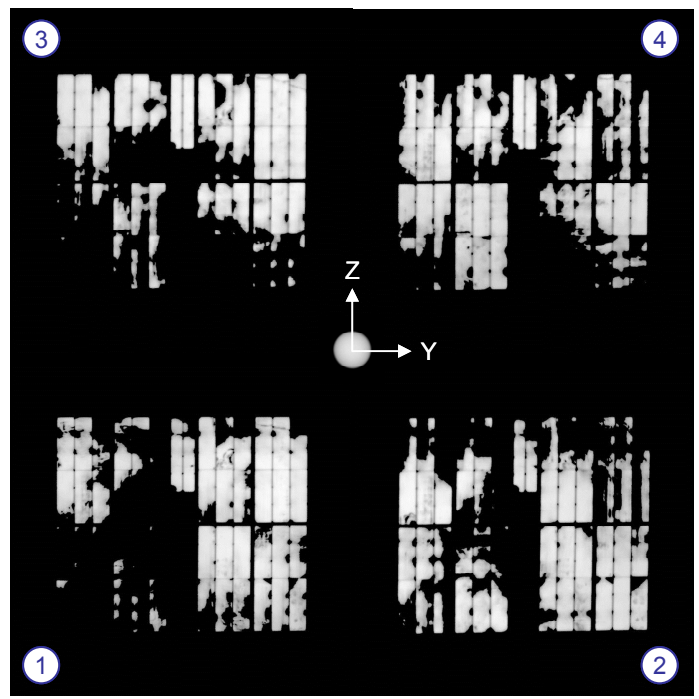

Figure 11: Same illustration as in Figure 10 with pre-defined canting errors added to heliostat E06.

The final results obtained with the reverse ray-tracing optimization algorithm $(\S 0.8)$ are illustrated in Figure 12, showing:

- The measured slopes errors along the $\mathrm{Y}$ and $\mathrm{Z}$ axes over the whole surface of the heliostat in its initial state in panels (a) and (b) respectively,

- Slopes errors along $\mathrm{Y}$ and $\mathrm{Z}$ axes after introduction of canting errors, in panels (c) and (d),

- Differences between previous slopes errors along $\mathrm{Y}$ and $\mathrm{Z}$ axes, in panels (e) and (f). For all points $\mathrm{P}$ at the heliostat surface, such a difference are only computed if the intersection mask function $M(\mathrm{P})=M_{\mathrm{I}}(\mathrm{P}) \cap M_{\mathrm{E}}(\mathrm{P})$ is non null, with $M_{\mathrm{I}}(\mathrm{P})$ and $M_{\mathrm{E}}(\mathrm{P})$ being the mask functions of the heliostat in the initial state and with added misalignments respectively. Otherwise the result is left blank. 
Detailed views of the $\mathrm{Y}$ and $\mathrm{Z}$ slopes difference maps for the two misaligned modules of the heliostat are also reproduced in Figure 13. They show that valid data is only available on sparse areas, particularly for module 2 . Moreover their general aspect looks noisy and dotted with missing points $\mathrm{P}$ that were eliminated during the optimization process because the achieved cost function $C F$ was superior to the authorized threshold value $t_{\mathrm{CF}}$ (the latter was set to 0.1 empirically). Nevertheless, Figure 13-c and 12-d clearly reveal slopes difference offsets directly linked to the canting errors that were introduced in elevation, while similar offsets are not visible on Figure 13-a and 12-b that can only reveal azimuth canting errors. Taking the average values of the Z-slopes offsets over the whole surface of the modules then gives a direct estimation of the added canting errors $h_{2}$ and $h_{3}$. The measured values for $h_{2}$ and $h_{3}$ are found to be -1.57 and +0.80 mrad respectively (see Table 1 ), which correspond the measurement errors equal to $\left|\delta h_{2}\right|=0.08$ and $\left|\delta h_{3}\right|=0.24 \mathrm{mrad}$. Such numbers are compatible with the estimated precision of the manual screws $(0.27 \mathrm{mrad}$, see text above). Moreover, the higher value of $\delta h_{3}$ may be biased by the presence of a local surface error clearly visible on module 3 and marked by red circles in Figure 13-b and 12-d. Also of interest are the measured values for azimuthal canting errors $a_{2}$ and $a_{3}$; which, let us remember, are nonexistent since their adjustment screws were untouched. From Table 1, the uncertainties are estimated as $\left|\delta a_{2}\right|=0.08$ and $\left|\delta a_{3}\right|=0.04 \mathrm{mrad}$, which is in agreement with the initial requirement of $0.14 \mathrm{mrad}$.

Table 1 finally provides a complete statistic analysis of the canting and surface slopes errors for each of the nine modules constituting the heliostat. In the "Spatial coverage" column is indicated the ratio of the useful area of the module where the $\mathrm{Y}$ or $\mathrm{Z}$ slopes could actually be extracted (i.e. those areas where the built mask function $M(\mathrm{P})$ is non null), with respect to the whole surface of the module. The columns "Mean", "PTV" and "RMS" respectively give the average, root mean square and peak-to-valley characteristics of the slopes error over the surface of each module. These numbers are finally averaged over the whole heliostat surface in the rows labelled as "Average". Table 1-A, 1-B and 1-C respectively correspond to the cases of heliostat in initial state, then with additional canting errors, and their difference. For cases A and B, the PTV and RMS numbers represent the residual surface slopes errors of the modules, while their mean values should be taken as their canting errors. Disregarding modules 2 and 3 that were discussed previously and module 1 where the spatial coverage is deemed insufficient, it seems that heliostat E06 has been fairly aligned since most canting errors are well below $0.5 \mathrm{mrad}$, excepting module 8 (azimuthal error around $-0.6 \mathrm{mrad}$ ) and the complementary module 9 (elevation error around $0.5 \mathrm{mrad}$ ). Assuming that the shape of the heliostat was stable between the two measurements that both took place in less than two minutes, only differential canting errors should be present in case C. Then it can be noted that:

- High PTV numbers (typically around 2 mrad) are found even for case C. They are mostly due to a few isolated peaks or dips located at the edges of the mask functions and hardly visible in the Figures. These points could have been eliminated by applying a lower threshold $t_{\mathrm{CF}}$ during the optimisation process, eventually at the risk of decreasing the spatial coverage at the surface of some heliostat modules.

- Nevertheless, all RMS numbers remain quite low and their averaged values over the whole heliostat surface can be taken as reliable indicators of the achieved measurement accuracy of the method. The latter are found to be 0.22 and $0.15 \mathrm{mrad}$ for $\mathrm{Y}$ and $\mathrm{Z}$ slopes errors respectively. From these values and the estimated uncertainties on the introduced canting errors on modules 2 and 3, one may finally conclude that the achieved measurement accuracy of our current test setup is around $0.2 \mathrm{mrad}$. This measurement uncertainty is estimated from the RMS error of the reconstructed slopes for the difference case of Table 1.C, based on data (pixels) shown in Figures 12-e and 12-f. Each pixel where the reconstruction is possible provides slopes values used to compute the RMS deviation of the canting error for each 
module. The average RMS deviation is then estimated on the entire heliostat from all illuminated pixels on the cameras, thus thousands of pixels enter the computation (e.g. 3200 and 16000 pixels for modules 2 and 3 respectively). Assuming the error distribution is Gaussian, the standard deviation (corresponding to RMS deviation) is considered a good approximation of the canting error experimental uncertainty.

Y-slopes
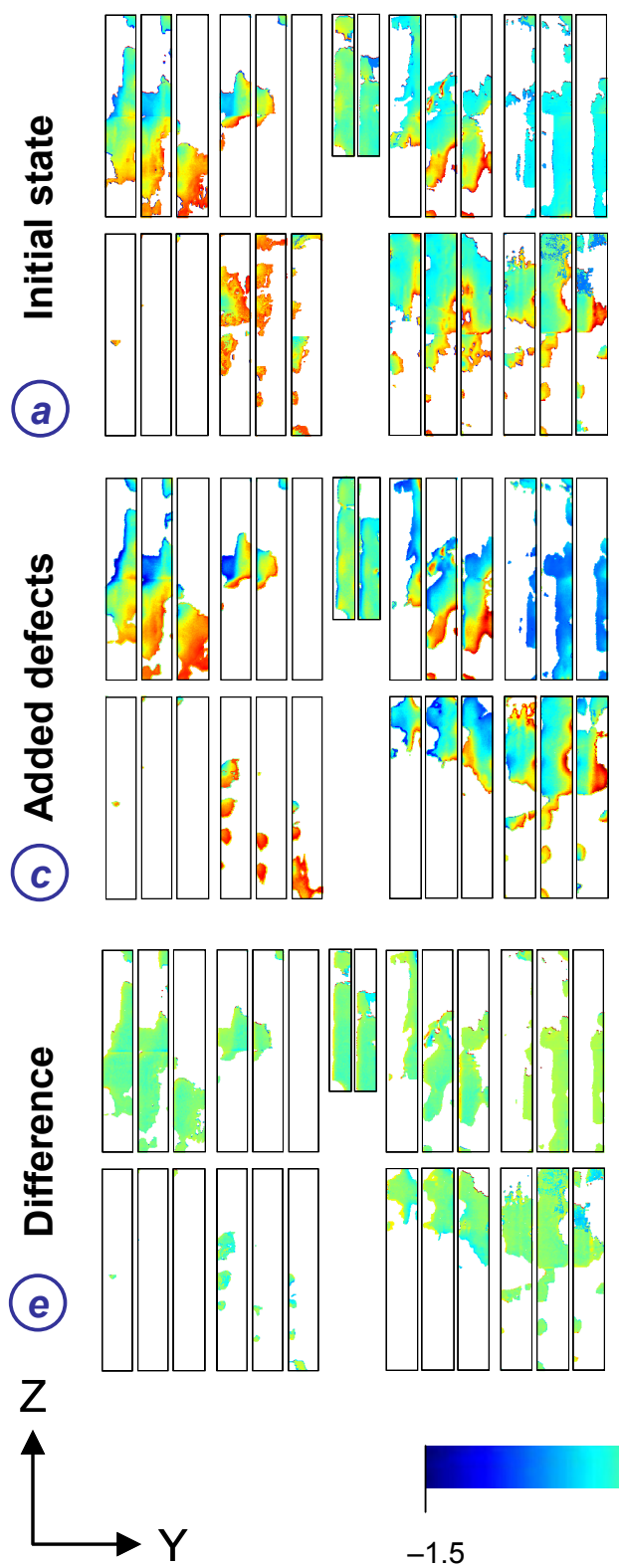
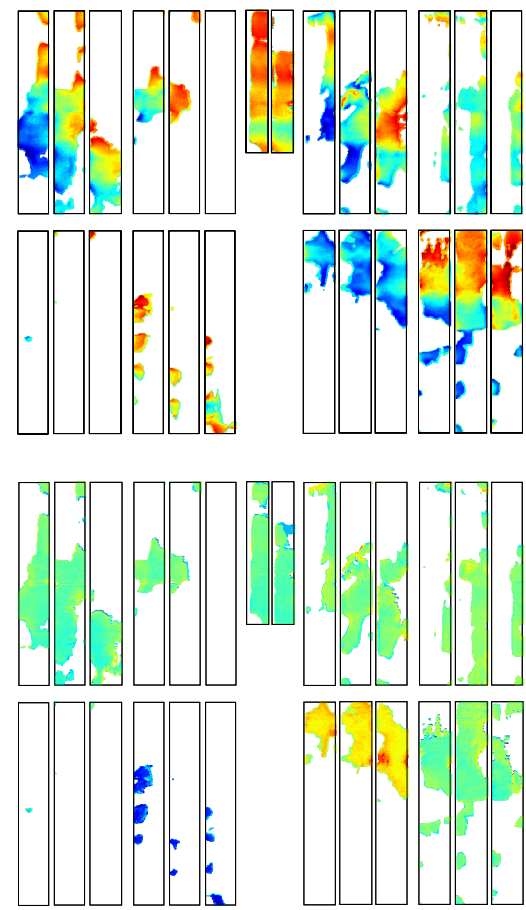

(d)

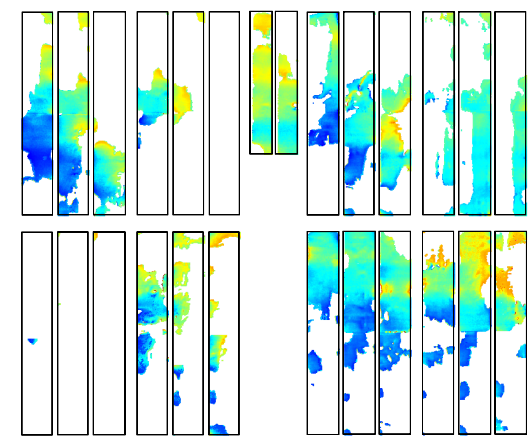

(b)
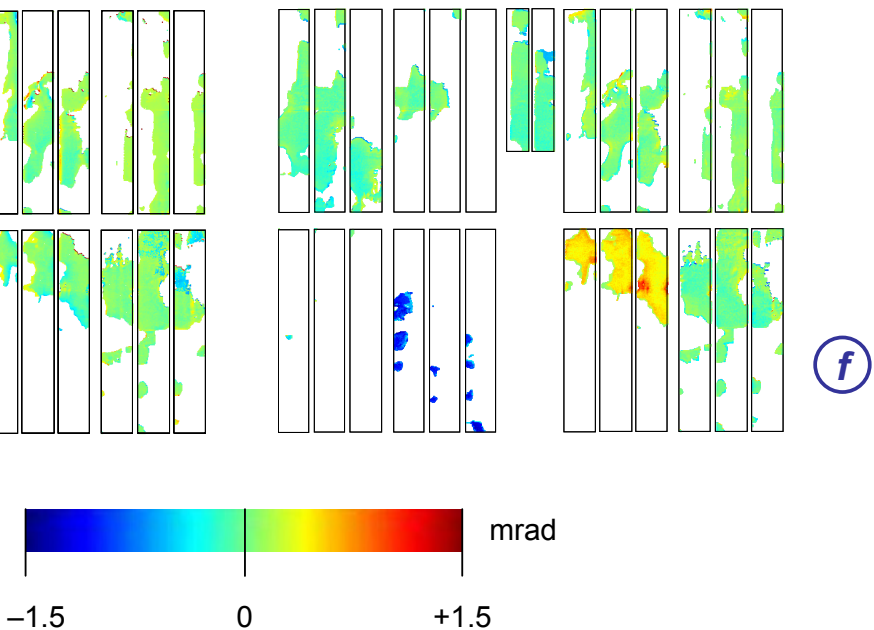

Figure 12: Measured slopes errors on heliostat E06 and their difference maps. (a) Y-slopes, heliostat in initial state. (b) Z-slopes, heliostat in initial state. (c) Y-slopes, heliostat with predetermined canting errors. (d) Z-slopes, heliostat with pre-determined canting errors. (e) Yslopes differences. (f) Z-slopes differences. 


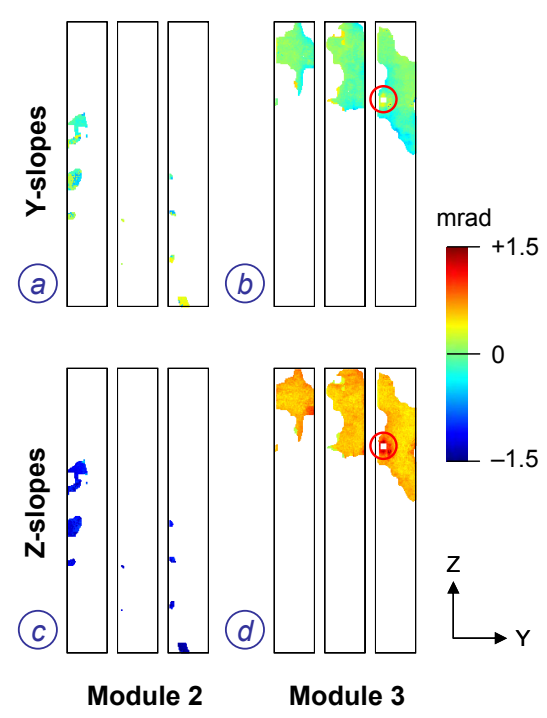

Figure 13: .Detailed views of slopes errors differences. (a) X-slopes, module 2 of heliostat E06. (b) X-slopes, module 3. (c) Y-slopes, module 3. (d) Y-slopes, module 3.

\subsection{Shape reconstruction}

This set of measurements was carried out on March 2019, the 15th between $14 \mathrm{~h} 25$ and $15 \mathrm{~h} 55$ local time, on a windy day with solar angles $\left(a_{\mathrm{S}}, h_{\mathrm{S}}\right)$ varying between $(+40.7,+37.2)$ and $(+43.5,+35.9)$ degs. Only the module 3 was misaligned with the same canting error as in section 0 . The measured value for $h_{3}$ was $0.70 \mathrm{mrad}$, leading to a measurement accuracy $\left|\delta h_{3}\right|$ $=0.19 \mathrm{mrad}$ compatible with the previous experiment. More interestingly, this new experiment offers a good opportunity to test the robustness of the reflective surface of the heliostat against wind and gravity loads. It is thus necessary to reconstruct the shape error function $\Delta(\mathrm{P})$ of the heliostat from its measured slopes $\partial \Delta(\mathrm{P}) / \partial y$ and $\partial \Delta(\mathrm{P}) / \partial z$. For that purpose, there exists a variety of algorithms that were developed for adaptive optics applications in the last decades (see e.g. Ref. [19]). Here was selected the iterative Fourier transform algorithm [20] for its ability to perform efficient shape reconstruction over highly discontinuous pupil masks in a reasonable period of time. The main numerical results are illustrated in Figure 14 and the mean, PTV and RMS statistics are indicated in Table 2 for each individual module, again excepting module 1 where the spatial coverage is insufficient.

The analysis of the shape error maps is a bit more difficult than for slopes error maps. It may firstly be noticed that these errors are mild since their PTV numbers never exceed $1 \mathrm{~mm}$. One may not forget however that a large fraction of the reflective surface could not be measured, which suggests that the errors are larger in these areas. Moreover, the employed algorithms for slopes and surface reconstruction are not sensitive to "piston" errors (i.e. shifts along X-axis) affecting the individual modules. However such errors usually do not degrade the concentrating performance significantly.

Secondly, no noise seems to be present in the reconstructed shape error maps, or at least it should remain at a much lower level than in slopes error maps. This is a well-known favourable effect of the surface reconstruction procedure establishing a best compromise between slopes errors along two orthogonal directions. Thus reasonable confidence may be given to the reconstructed surface even if no absolute reference is available, and consequently the effects of wind and gravity loads cannot be disentangled. 
Table 1: Numerical results of slopes error measurements obtained for: A) Heliostat in initial state. B) Heliostat with canting errors added. C) Difference between both states.

\section{A. Initial state}

\begin{tabular}{|c|c|c|c|c|c|c|c|}
\hline \multirow{2}{*}{ Module number } & \multirow{2}{*}{$\begin{array}{l}\text { Spatial } \\
\text { coverage }\end{array}$} & \multicolumn{3}{|c|}{ Y slopes } & \multicolumn{3}{|c|}{$Z$ slopes } \\
\hline & & Mean (mrad) & PTV (mrad) & RMS (mrad) & Mean (mrad) & PTV (mrad) & RMS (mrad) \\
\hline 1 & 0.000 & 1.066 & 0.041 & 0.020 & -1.076 & 0.028 & 0.014 \\
\hline 2 & 0.110 & 0.581 & 2.785 & 0.471 & 0.013 & 2.568 & 0.644 \\
\hline 3 & 0.380 & -0.118 & 2.667 & 0.378 & -0.154 & 2.629 & 0.410 \\
\hline 4 & 0.261 & -0.086 & 2.813 & 0.460 & 0.244 & 3.079 & 0.610 \\
\hline 5 & 0.304 & 0.157 & 2.965 & 0.526 & -0.231 & 2.603 & 0.586 \\
\hline 6 & 0.062 & -0.060 & 2.417 & 0.498 & 0.272 & 2.364 & 0.516 \\
\hline 7 & 0.237 & -0.079 & 2.840 & 0.456 & -0.051 & 3.087 & 0.531 \\
\hline 8 & 0.201 & -0.532 & 1.520 & 0.122 & -0.021 & 2.335 & 0.209 \\
\hline 9 & 0.498 & -0.126 & 1.829 & 0.217 & 0.474 & 1.618 & 0.392 \\
\hline Average & & 0.089 & 2.209 & 0.350 & -0.059 & 2.257 & 0.435 \\
\hline
\end{tabular}

\section{B. Added defects}

\begin{tabular}{|c|c|c|c|c|c|c|c|}
\hline \multirow{2}{*}{ Module number } & \multirow{2}{*}{$\begin{array}{l}\text { Spatial } \\
\text { coverage }\end{array}$} & \multicolumn{3}{|c|}{ Y slopes } & \multicolumn{3}{|c|}{$Z$ slopes } \\
\hline & & Mean (mrad) & PTV (mrad) & RMS (mrad) & Mean (mrad) & PTV (mrad) & RMS (mrad) \\
\hline 1 & 0.000 & -0.166 & 0.112 & 0.046 & 1.141 & 0.083 & 0.034 \\
\hline 2 & 0.041 & 0.802 & 1.692 & 0.308 & 0.521 & 1.966 & 0.370 \\
\hline 3 & 0.195 & -0.246 & 2.089 & 0.394 & -0.604 & 1.434 & 0.252 \\
\hline 4 & 0.309 & 0.019 & 2.326 & 0.395 & 0.288 & 2.540 & 0.586 \\
\hline 5 & 0.306 & 0.252 & 2.689 & 0.548 & -0.124 & 2.647 & 0.541 \\
\hline 6 & 0.062 & -0.023 & 2.299 & 0.558 & 0.293 & 2.403 & 0.475 \\
\hline 7 & 0.236 & -0.046 & 2.638 & 0.573 & -0.044 & 2.639 & 0.573 \\
\hline 8 & 0.143 & -0.723 & 1.237 & 0.163 & -0.166 & 1.738 & 0.252 \\
\hline 9 & 0.547 & -0.050 & 1.723 & 0.211 & 0.551 & 1.768 & 0.320 \\
\hline Average & & -0.020 & 1.867 & 0.355 & 0.206 & 1.913 & 0.378 \\
\hline
\end{tabular}

\section{Difference}

\begin{tabular}{|l|l|lll|lll|}
\hline \multirow{2}{*}{ Module number } & \multirow{2}{*}{$\begin{array}{l}\text { Spatial } \\
\text { coverage }\end{array}$} & \multicolumn{3}{|c|}{ Y slopes } & \multicolumn{3}{c|}{ Z slopes } \\
\cline { 3 - 7 } & & Mean (mrad) & PTV (mrad) & RMS (mrad) & Mean (mrad) & PTV (mrad) & RMS (mrad) \\
\hline 1 & 0.000 & & & & -1.571 & 1.396 & 0.210 \\
2 & 0.017 & -0.085 & 1.884 & 0.404 & 0.796 & 1.874 & 0.163 \\
3 & 0.181 & -0.044 & 2.501 & 0.203 & 0.069 & 1.555 & 0.166 \\
4 & 0.253 & -0.040 & 2.148 & 0.328 & -0.042 & 1.339 & 0.146 \\
5 & 0.283 & -0.086 & 1.849 & 0.151 & 0.063 & 1.191 & 0.128 \\
6 & 0.057 & -0.041 & 2.337 & 0.199 & 0.127 & 1.707 & 0.144 \\
7 & 0.200 & 0.000 & 1.981 & 0.210 & 0.183 & 1.624 & 0.120 \\
8 & 0.138 & 0.218 & 1.566 & 0.098 & -0.045 & 1.151 & 0.155 \\
9 & 0.470 & -0.116 & 1.726 & 0.198 & -0.053 & 1.480 & 0.154 \\
\hline Average & & -0.024 & 1.999 & 0.224 & & \\
\hline
\end{tabular}


Thirdly, careful inspection of certain modules in Figure 14-a and 13-b is instructive. Tilts in nearly pure elevation (i.e. around Y-axis) are visible on modules 3, 7 and 9, which is in agreement with the "mean" numbers listed in Table 1 . The same tendency can be observed on module 4 with a slight additional component in azimuth (around Z-axis). Other heliostat modules such as numbers 2 and 5 seem to exhibit higher-order types of shape distortion.

Finally, Figure 14-c reveals the shape deformation of heliostat E06 between both times of measurement. For a rigid body the difference map between both states should display uniform colours over the surface of each individual module (obviously excepting module 3 where the canting error was introduced). This is achieved for some of them, e.g. module 6 and the complementary module 9 that look rather stable. Some other, particularly the module 2, exhibit noticeable deformations that are confirmed by high PTV and RMS numbers in Table 2-C. Such distortions may find their origin in wind or gravity loads varying between both measurement times.

To conclude, it seems that the backward gazing method with four cameras demonstrated its ability to characterize the surface distortion of a focusing heliostat in sun-tracking mode, even if the global measurement accuracy of the method needs to be improved as discussed in the following section.

\subsection{Potential improvements}

The measurement accuracy achieved by our experiment has been estimated around $0.2 \mathrm{mrad}$ RMS (see section 0), which is equal to twice our initial requirement of $0.1 \mathrm{mrad}(\S 0)$. An analysis of the main experimental error sources is presented below, as well as possible ways of mitigation.

1) The most important error is originating from the cameras affected by a significant amount of noise, as revealed by careful inspection of the acquired Sun and heliostat images $B_{0}^{n}(v, w)$ and $B_{i j}^{n^{\prime}}(P)$, whose effects are also visible in Figure 5, 11 and 12. This noise was roughly estimated to 3-4 \% PTV. This is coherent with Ref. [9], where numerical simulations in section 3.C.5 showed that a white noise with $1 \%$ amplitude results in WFE slopes errors around $0.1 \mathrm{mrad}$, thus surface slopes errors of $0.05 \mathrm{mrad}$. Such image noise is clearly due to an insufficient number of simultaneously captured images, currently equal to $\mathrm{N}=\mathrm{N}^{\prime}=5$. To improve the reconstruction accuracy, a much larger number of images should be recorded and averaged from each camera. This number can be increased by extending the switch bandwidth and using Ethernet cables having larger image memory.

2) Another difficulty arises from the employed image acquisition software, which does not offer the capacity of real-time image visualisation. Practically, it prevents us to check the sharpness of the images at the surface of the heliostat during acquisition: it follows that the focusing adjustment of the camera had to be performed with the bare camera lens, before manual installation of the optical densities with a risk of slightly modifying the focus adjustment. This issue can be addressed by changing the acquisition software or the cameras themselves so that they could display living images of the heliostat during acquisition, ideally with the help of a remotely-controlled focusing adjustment device. 

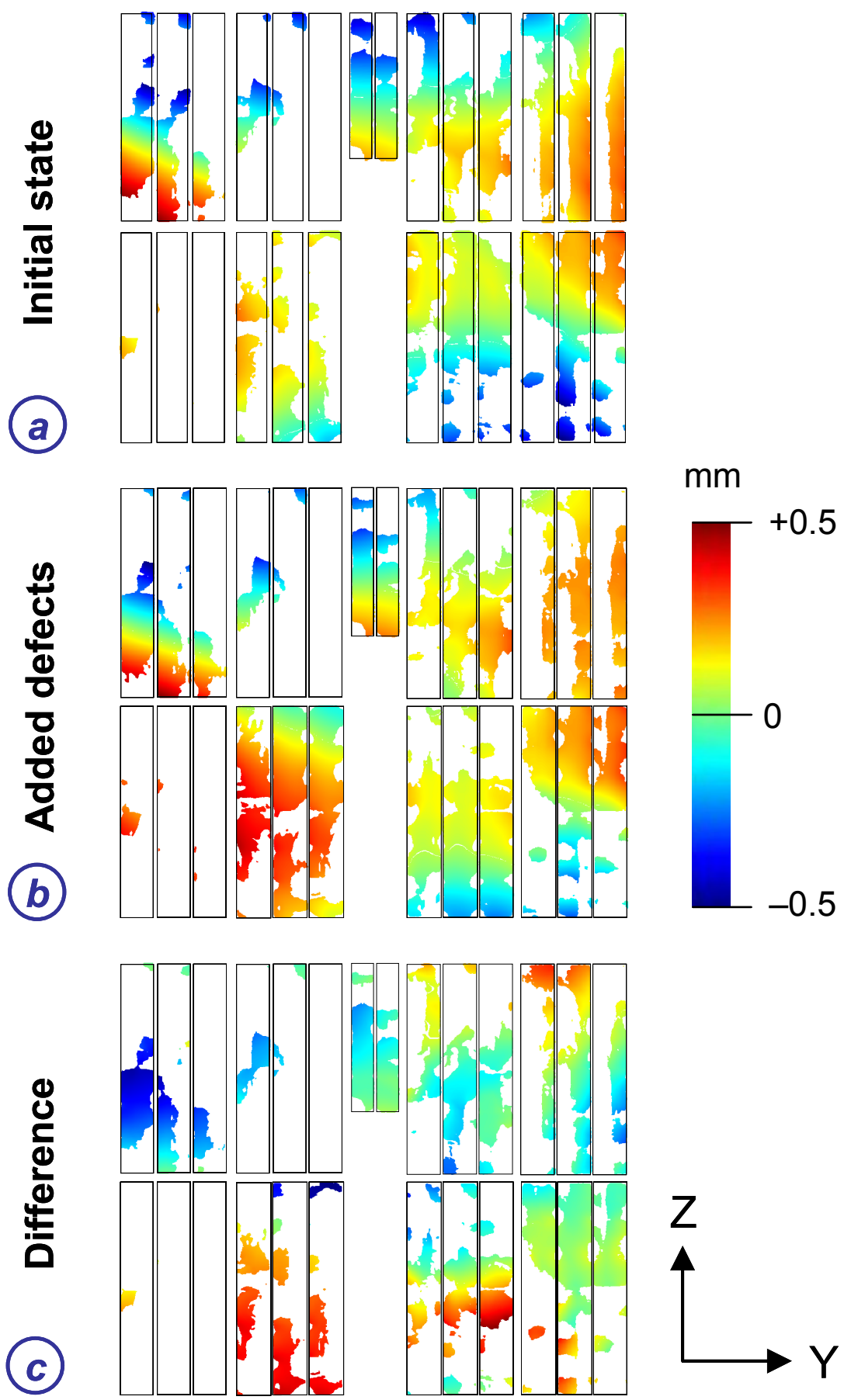

Figure 14: Shape reconstruction for heliostat E06. (a) Heliostat in initial state. (b) Heliostat with pre-determined canting error on module 3. (c) Shape errors difference. 
Table 2: Numerical results of shape reconstruction procedure obtained for: A) Heliostat in initial state. B) Heliostat with canting error added to module 3. C) Difference between both states.

\section{A. Initial state}

\begin{tabular}{lllll}
\cline { 2 - 5 } Module number & \multicolumn{3}{c}{ Shape errors } \\
\cline { 2 - 5 } & Coverage & Mean $(\mathrm{mm})$ & PTV $(\mathrm{mm})$ & RMS $(\mathrm{mm})$ \\
\hline 1 & 0.000 & & & \\
2 & 0.110 & 0.011 & 0.482 & 0.116 \\
3 & 0.380 & -0.112 & 0.661 & 0.117 \\
4 & 0.261 & -0.239 & 0.819 & 0.190 \\
5 & 0.304 & -0.133 & 0.816 & 0.199 \\
6 & 0.062 & -0.040 & 0.409 & 0.074 \\
7 & 0.237 & -0.072 & 0.563 & 0.106 \\
8 & 0.201 & -0.063 & 0.506 & 0.096 \\
9 & 0.498 & 0.025 & 0.729 & 0.204 \\
\hline Average & & -0.078 & 0.623 & 0.138 \\
\hline
\end{tabular}

\section{B. Added defects}

\begin{tabular}{lllll}
\cline { 2 - 5 } Module number & \multicolumn{3}{c}{ Shape errors } \\
\cline { 2 - 5 } & Coverage & Mean $(\mathrm{mm})$ & PTV $(\mathrm{mm})$ & RMS $(\mathrm{mm})$ \\
\hline 1 & 0.000 & & & \\
2 & 0.041 & 0.059 & 0.363 & 0.067 \\
3 & 0.195 & -0.079 & 0.883 & 0.168 \\
4 & 0.309 & -0.151 & 0.774 & 0.183 \\
5 & 0.306 & -0.099 & 0.655 & 0.150 \\
6 & 0.062 & -0.023 & 0.496 & 0.082 \\
7 & 0.236 & -0.136 & 0.705 & 0.130 \\
8 & 0.143 & -0.069 & 0.597 & 0.127 \\
9 & 0.547 & -0.027 & 0.987 & 0.263 \\
\hline Average & & -0.066 & 0.683 & 0.146 \\
\hline
\end{tabular}

\section{Difference}

\begin{tabular}{lllll}
\cline { 2 - 5 } Module number & \multicolumn{3}{c}{ Shape errors } \\
\cline { 2 - 5 } & Coverage & Mean $(\mathrm{mm})$ & PTV $(\mathrm{mm})$ & RMS $(\mathrm{mm})$ \\
\hline 1 & 0.000 & & & \\
2 & 0.017 & 0.081 & 0.658 & 0.157 \\
3 & 0.181 & 0.095 & 0.819 & 0.189 \\
4 & 0.253 & 0.079 & 0.325 & 0.034 \\
5 & 0.283 & 0.028 & 0.327 & 0.068 \\
6 & 0.057 & 0.020 & 0.121 & 0.016 \\
7 & 0.200 & -0.058 & 0.279 & 0.059 \\
8 & 0.138 & 0.010 & 0.281 & 0.054 \\
9 & 0.470 & -0.069 & 0.409 & 0.078 \\
\hline Average & & 0.023 & 0.403 & 0.082 \\
\hline
\end{tabular}


3) The experimental setup located behind the passive target could also be improved. Currently, the four cameras are fixed on two rails directly attached to target plane (see Figure 8) that undergoes thermal expansion when it is illuminated by the heliostats. Possible image instabilities of vignetting effects may occur and alter the accuracy of the slopes reconstruction process. The implementation of mechanical supports not connected to the target is desirable. Here again, real-time images visualisation would be helpful to detect and prevent such effects.

4) Regarding image processing algorithms, one may think that their development and validation are already well advanced. We noted however that the "parallelogram approximation" used to rectify the images of the heliostats (see $\S 0.3$ ) suffers from a lack of precision, especially for pixels located near the edges of the mask area. Although slight, this effect is visible on certain heliostat modules in Figure 12. For achieving image superposition at the sub-pixel level over the whole surface of the heliostat, it should be necessary to correct the small pyramidal effect that remains in the acquired images. This modification will be implemented in the next version of the image processing software.

5) One last but very important issue consists in improving the spatial coverage of the method in order to characterize the entire surface of the heliostat. Actually, the limited spatial coverage in Figures 11 to 13 result from the combination of large slopes errors (typically higher than $1 \mathrm{mrad}$ ) and a limited number of cameras separated by too short distances. It has already been demonstrated that using more numerous cameras arranged in a square geometry allows overcoming this limit [13]. Alternatively, a much lesser number of cameras (typically 8 or 10) could be arranged along the Z'-axis of the target plane and combined with a suntracking program allowing the heliostat to scan the target plane perpendicularly to the line of cameras for tracking errors monitoring. This point will be the scope of future studies.

In conclusion, important modifications of the experimental setup are required in order to attain our initial goal of $0.1 \mathrm{mrad}$ measurement accuracy. Nevertheless, considering the already achieved performance (around $0.2 \mathrm{mrad}$ ) and the improvement possibilities listed above gives to us reasonable confidence in reaching the final requirement in the near future.

\section{Summary}

In this paper were presented the first experimental results obtained with the four cameras backward gazing method for measuring the opto-mechanical errors of solar concentrating surfaces in operation. The method consists in combining the images simultaneously acquired by four cameras placed near the solar receiver in order to reconstruct the slopes errors of the mirrors digitally. A great effort was undertaken to adapt the reconstruction procedures described in previous publications to the case of real images acquired by the cameras. The experiment has been conducted on a focusing heliostat of the experimental solar tower plant THEMIS in Targassonne (France). To our knowledge, it produced the first slopes error measurements ever made on a heliostat in sun-tracking mode with measurement accuracy around $0.2 \mathrm{mrad}$. The capacity of characterizing shape distortions resulting from wind or gravity loads has also been investigated. The main experimental error sources have been analysed, as well as possible ways of mitigation giving reasonable hope in reaching an ultimate measurement accuracy of $0.1 \mathrm{mrad}$. 


\section{Funding}

This project has received funding from the European Union's Horizon 2020 research and innovation programme under grant agreement No 727762, Next-CSP project and was supported by the French "Investments for the future" ("Investissementsd'Avenir") programme managed by the National Agency for Research (ANR) under contracts ANR-10-LABX-22-01 (Labex SOLSTICE) and ANR-10-EQPX-49 (Equipex SOCRATE).

\section{Disclosures}

The authors declare no conflicts of interest.

\section{References}

1. J. Xiao, X. Wei, Z. Lu, W. Yu, H .Wu, "A review of available methods for surface shape measurement of solar concentrator in solar thermal power applications," Renewable and Sustainable Energy Reviews vol. 16, p. 2539-2544 (2012).

2. L. Ren, X. Wei, Z. Lu, W. Yu, W. Xu, Z. Shen,"A review of available methods for the alignment of mirror facets of solar concentrator in solar thermal power system", Renewable and Sustainable Energy Reviews vol. 32, p. 76-83 (2014).

3. C.A. Arancibia-Bulnes, M.I Peña-Cruz, A. Mutuberría, R. Díaz-Uribe, M. Sánchez-González, "A survey of methods for the evaluation of reflective solar concentrator optics," Renewable and Sustainable Energy Reviews vol. 69, p. 673-684 (2017).

4. K. Pottler, E. Lüpfert, G. H. Johnston, and M. R. Shortis, "Photogrammetry: a powerful tool for geometric analysis of solar concentrators and their components," in ASME International Solar Energy Conference (American Society of Mechanical Engineers, 2004), p. 719-726.

5. T. A. Moss, R. B. Diver, "Practical field alignment of parabolic trough solar concentrators," J. Sol. Energy Eng. vol. 129 , p. 153-159 (2007).

6. S. Ulmer, T. März, C. Prahl, W. Reinalter, B. Belhomme, "Automated high resolution measurement of heliostat slope errors," Solar Energy vol. 85, p. 681-687 (2011).

7. C. E. Andraka, J. Yellowhair, K. Trapeznikov, J. Carlson, B. Myer, B. Stone, K. Hunt, "AIMFAST: An alignment tool based on fringe reflection methods applied to dish concentrators," Journal of Solar Energy Engineering vol. 133, $\mathrm{n}^{\circ} 031018$ (2011).

8. F. Henault, C. Royere, "Concentration du rayonnement solaire: analyse et évaluation des réponses impulsionelles et des défauts de réglage de facettes réfléchissantes”, J. Optics vol. 20, n5 (1989).

9. M. Coquand, F. Hénault, C. Caliot, "Backward-gazing method for measuring solar concentrators shape errors," Applied Optics vol. 56, p. 2029-2037 (2017).

10. M. Coquand, C. Caliot, F. Hénault, "Tracking and shape errors measurement of concentrating heliostats," Proceedings of the SPIE vol. 10379, ${ }^{\circ} 103790 \mathrm{~N}-1$ (2017).

11. M. Coquand, F. Hénault, C. Caliot, "Numerical identification of mirror shapes with the backward-gazing method using an actual solar profile," Proceedings of SolarPaces 2017, AIP conferences series vol. 2033, $\mathrm{n}^{\circ} 040010(2018)$.

12. M. Coquand, "Méthode de rétrovisée pour la caractérisation de surfaces optiques dans une installation solaire à concentration," Thèse de Docteur en Sciences, Université de Perpignan Via Domitia (2018).

13. F. Hénault, M. Coquand, P.-H. Defieux, C. Caliot, "Sun backward gazing method with multiple cameras for characterizing solar concentrators," Solar Energy vol. 166, p. 103-114 (2018).

14. P.H. Defieux, C. Caliot, F. Hénault, "Hybrid Optical Method for Characterizing a Heliostat Field in a Concentrated Solar Power Plant," SolarPACES 2019 conference, to be published in AIP conferences series.

15. P. Jose, "The flux through the focal spot of a solar furnace," Solar Energy vol. 1, p. 19-22 (1957).

16. F. Hénault, "Concentration du rayonnement solaire par simple et double réflexion: Contribution aux méthodes de réglage et de contrôle des surfaces réflectrices à facettes," PhD Thesis, Université Paris XI Orsay (1987).

17. A. Sanchez-González, C. Caliot, A. Ferrière, D. Santana, "Determination of heliostat canting errors via deterministic optimization," Solar Energy vol. 150, p. 136-146 (2017).

18. F. Hénault, "Fast computation of solar concentrating ratio in presence of opto-mechanical errors," Solar Energy vol. 112, p. 183-193 (2015)

19. W. H. Southwell, "Wave-front estimation from wave-front slope measurements," J. Opt. Soc. Am. vol. 70, p. 998-1006 (1980).

20. F. Roddier, C. Roddier, "Wavefront reconstruction using iterative Fourier transforms," Applied Optics vol. 30, p 1325-1327 (1991). 


\section{Appendix A. Image derotation algorithm}

For each median and radiometrically calibrated image $B_{i j}(P)$ the following processing steps are applied. The whole procedure is illustrated in Figure A1.

1. Starting from the original image $B_{i j}(P)$ depicted in Figure A1-a, a relative brightness threshold factor $t_{\mathrm{B}}$ is firstly applied: $B_{i j}(P)$ is set to zero if it is lower than $t_{\mathrm{B}}$, which is typically taken equal to 0.5 or to the parameter $a$ when the generalized Jose formula in Eq. 6 is used.

2. Denoting $y_{\mathrm{H}}$ and $z_{\mathrm{V}}$ the horizontal and vertical axes of the captured image, the gradients $\partial B_{i j}(P) / \partial y_{H}$ and $\partial B_{i j}(P) / \partial z_{V}$ are computed as illustrated in Figures A1-b and A1-c.

3. The previous image gradients are Fourier transformed as $\hat{B}_{Y i j}(\mu, v)=F T\left[\partial B_{i j}(P) / \partial y_{H}\right]$ and $\hat{B}_{Z i j}(\mu, v)=F T\left[\partial B_{i j}(P) / \partial z_{V}\right]$. Pictures of these spectra in logarithmic scale are shown in Figures A1-d and A1-e. As predicted from theory, most of their power lays along or near to two axes that correspond to the $\mathrm{Y}$ and $\mathrm{Z}$ heliostat contour, and are also enforced by the contours of the individual modules of the heliostat.

4. Both functions $\hat{B}_{Y_{i j}}(\mu, v)$ and $\hat{B}_{Z_{i j}}(\mu, v)$ are normalized by their maximal values at the origin $\hat{B}_{Y_{i j}}(0,0)$ and $\hat{B}_{Z_{i j}}(0,0)$. A threshold factor $t_{\mathrm{S}}$ is applied, typically equal to 0.1 .

5. Two histograms $H_{Y i j}(\theta)$ and $H_{Z i j}(\theta)$ of the power spectra distributions $\hat{B}_{Y_{i j}}(\mu, v)$ and $\hat{B}_{Y i j}(\mu, v)$ respectively, are built as function of the polar angle $\theta$ defined as $\theta=\arctan (v / \mu)$. After normalization by their maximal values, these histograms are depicted in Figure A1-f.

6. Both histograms are thresholded by a factor $t_{\mathrm{H}}$ typically equal to 0.4 .

7. For each histogram the location of their maximal values are determined with a centroiding algorithm. They are noted $\theta_{\mathrm{Y} i j}$ and $\theta_{Z i j}$ for $H_{\mathrm{Y} i j}(\theta)$ and $H_{Z i j}(\theta)$ respectively (see Figure A1-f).

8. Finally returning to the original images $B_{i j}(P)$, the rectangular contours of the heliostat are restored by using the coordinates transform matrix relationship:

$$
\left(\begin{array}{l}
y \\
z
\end{array}\right)=\left[\begin{array}{cc}
\cos \theta_{Y i l}+\sin \theta_{Y i l} \cos \left(\theta_{Z i l}-\theta_{Y i l}\right) & -\sin \theta_{Y i l}+\cos \theta_{Y i l} \cos \left(\theta_{Z i l}-\theta_{Y i l}\right) \\
\cos \theta_{Y i l} \sin \left(\theta_{Z i l}-\theta_{Y i l}\right) & -\sin \theta_{Y i l} \sin \left(\theta_{Z i l}-\theta_{Y i l}\right)
\end{array}\right]\left(\begin{array}{c}
y_{H} \\
z_{V}
\end{array}\right) \text {. }
$$

This last step is carried out using the WARP_TRI procedure provided with the Interactive Data Language (IDL ${ }^{\mathrm{TM}}$ ) software package. 


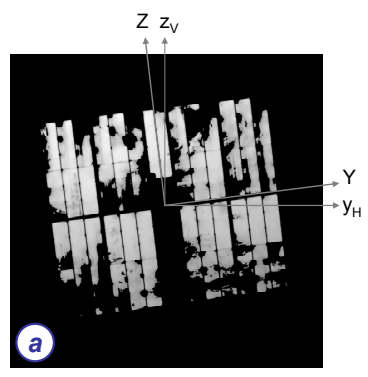

Z
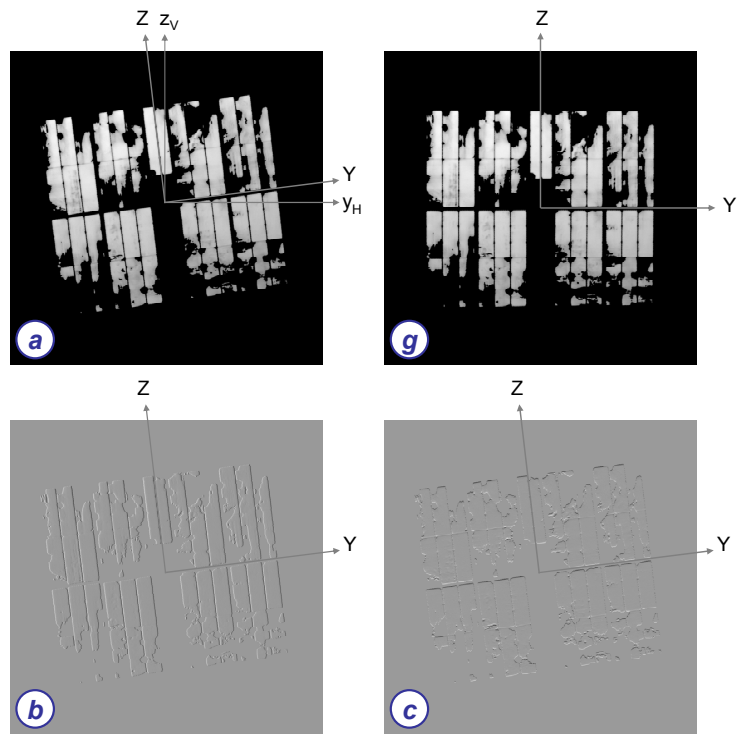

V

V
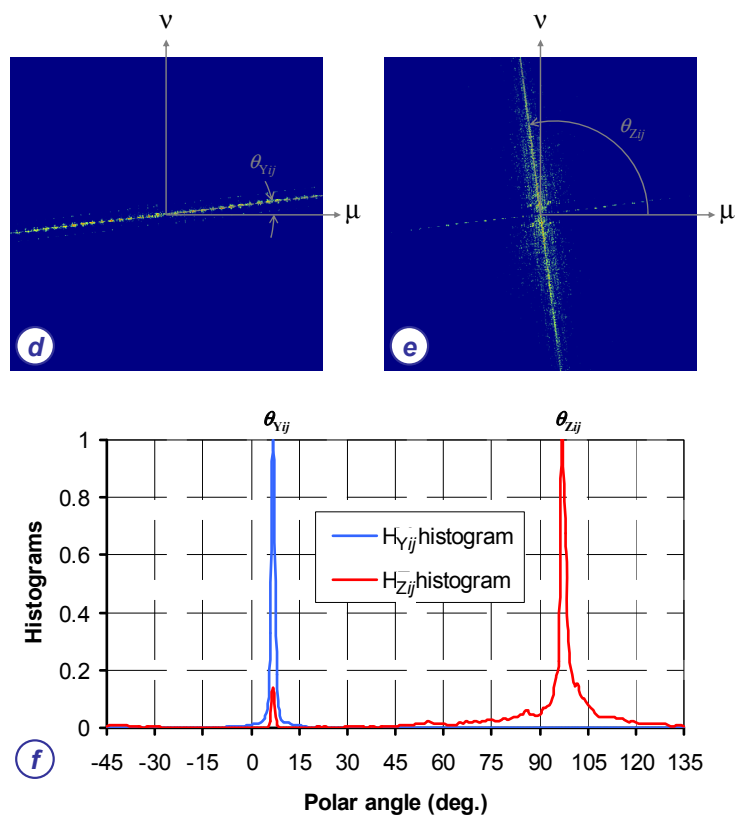

Figure A1: Illustrating the image derotation procedure. (a) Original rotated image. (b) and (c) Image gradients along the horizontal and vertical axes. (d) and (e) Fourier transforms of the image gradients in logarithmic scale. (f) Built histograms for evaluating $\theta_{Y}$ and $\theta_{Z}$ angles. (g) Final derotated and rectified image.

\section{Appendix B. Coarse and improved images registration}

For each rectified image $B_{i j}(P)$ the following processing steps are applied.

\section{B1. Coarse registration}


1. The $\mathrm{Y}$ and $\mathrm{Z}$ gradients $\partial B_{i j}(P) / \partial y$ and $\partial B_{i j}(P) / \partial z$ of the rectified image are firstly computed using the same procedure as in Appendix A. Their general aspect is quite similar as in Figures A1-b and A1-c, except that the contours of the heliostat modules are now parallel to the horizontal and vertical axes

2. A horizontal "contour function" $C_{Y i j}(y)$ is built by integrating the $\mathrm{Y}$ gradient map along the vertical Z-axis. Analytically, this operation writes as $C_{Y i j}(y)=\int_{-\infty}^{+\infty}\left[\partial B_{i j}(P) / \partial y\right] d z$. Practically, it is computed as the discrete sum $C_{Y i j}(y)=\sum_{m=-M_{p}}^{+M_{p}} \partial B_{i j}(P) / \partial y /\left(2 N_{p}+1\right)$, with $2 M_{p}+1$ and $2 N_{p}+1$ the number of useful pixels along the $\mathrm{Y}$ and $\mathrm{Z}$ axes respectively. Figure B1-a shows examples of horizontal contour functions computed from the images acquired by the four cameras simultaneously.

3. Similarly, vertical contour functions $C_{Z i j}(z)$ are computed by integrating the $Z$ gradients along the Y-axis, writing as $C_{Z i j}(z)=\int_{-\infty}^{+\infty}\left[\partial B_{i j}(P) / \partial z\right] d y$. Examples of vertical contour functions are shown in Figure B1-c.

4. Taking the absolute value $\left|C_{\mathrm{Y} i j}(y)\right|$ of the horizontal contour function, the locations of its first and last maxima are determined and noted $a_{i j}$ and $b_{i j}$. The same operation is performed on the function $\left|C_{Z i j}(z)\right|$, whose first and last maxima are found at $\mathrm{Z}$ coordinates $c_{i j}$ and $d_{i j}$.

5. Each image $B_{i j}(P)$ is finally shifted by the quantity $-\left(a_{i \mathrm{j}}+b_{i \mathrm{j}}\right) / 2$ along the Y-axis and by $-\left(c_{i \mathrm{j}}+d_{i \mathrm{j}}\right) / 2$ along the $\mathrm{Z}$-axis.

\section{B2. Improved registration}

After coarse registration of the four images $B_{i j}(P)$, one of them (e.g. $\left.B_{11}(P)\right)$ is defined as reference and the three other images must be recent red with respect to it. The following steps are applied to $B_{21}(P), B_{12}(P)$ and $B_{22}(P)$ :

6. From the contour function computed in section B1, the "horizontal" cross-correlation product $C C_{Y i j}(y)$ is evaluated as $C C_{Y i j}(y)=\int_{-\infty}^{+\infty} C_{Y i j}\left(y^{\prime}+y\right) C_{Y 11}\left(y^{\prime}\right) d y^{\prime}$, with indices $(i, j)=(2,1),(1,2)$ or $(2,2)$. Figure B1-b depicts the cross-correlation functions calculated from the horizontal contour functions shown in Figure B1-a.

7. Similarly, the vertical cross-correlation functions are computed as $C C_{Z i j}(z)=\int_{-\infty}^{+\infty} C_{Z i j}\left(z^{\prime}+z\right) C_{Z 11}\left(z^{\prime}\right) d z^{\prime}$. Figure B1-d shows the cross-correlation curves obtained from the vertical contour functions in Figure B1-c. 
8. A threshold factor $t_{\mathrm{CC}}$ is applied to the horizontal cross-correlation product $C C_{Y i j}(y)$, such that $C C_{Y_{i j}}(y)=C C_{Y i j}(y)-t_{C C}$ if $C C_{Y i j}(y)>t_{C C}$, and $C C_{Y i j}(y)=0$ otherwise. A similar operation is performed with the vertical cross-correlation product $C C_{Z i j}(z)$. A typical value for $t_{\mathrm{CC}}$ is 0.2 (see Figures B1-b and B1-d).

9. The location of the maximal value of the thresholded function $C C_{Y i j}(y)$ is determined with the help of a centroiding algorithm and noted $y_{C i j}$.

10. The same operation is repeated with the thresholded function $C C_{Z i j}(z)$, whose maximal value is found at the $z_{\mathrm{C} i j}$ coordinate.

11. For $(i, j)=(2,1),(1,2)$ or $(2,2)$, the image $B_{i j}(P)$ is finally shifted by the quantities $-y_{C i \mathrm{j}}$ and $-z_{C i \mathrm{j}}$ along the $\mathrm{Y}$ and $\mathrm{Z}$ axes respectively.

\section{Appendix C. Computing $\mathrm{A}$ and $\mathrm{H}$ angles}

Using the notations and coordinate systems defined in section 0 , the following computation steps are performed. Additional parameters are also employed:
$u_{\mathrm{S}}, v_{\mathrm{S}}, w_{\mathrm{S}}$
Direction cosines of Sun vector $\mathbf{S}_{\mathbf{0}}$ in $\mathrm{R}_{\mathrm{F}}$ reference frame
$x_{\mathrm{H}}, y_{\mathrm{H}}, z_{\mathrm{H}}$
Cartesian coordinates of the heliostat centre $\mathrm{O}$ in $\mathrm{R}_{\mathrm{F}}$ reference frame
$x_{\mathrm{R}}, y_{\mathrm{R}}, z_{\mathrm{R}}$ Cartesian coordinates of target plane centre $\mathrm{O}^{\prime}$ in $\mathrm{R}_{\mathrm{F}}$ reference frame
$a_{\mathrm{N}}, h_{\mathrm{N}}$
Azimuth and elevation angles of vector $\mathbf{N}_{\mathbf{0}}$ in $\mathrm{R}_{\mathrm{F}}$ reference frame
$u_{\mathrm{N}}, v_{\mathrm{N}}, w_{\mathrm{N}}$
Direction cosines of vector $\mathbf{N}_{\mathbf{0}}$ in $\mathrm{R}_{\mathrm{F}}$ reference frame

1. Knowing the date and acquisition time of measurement, the azimuth and elevation angles $a_{\mathrm{S}}$ and $h_{\mathrm{S}}$ of the Sun vector $\mathbf{S}_{\mathbf{0}}$ are firstly computed with the help of a Sun position calculator.

2. The Sun and target vectors $\mathbf{S}_{\mathbf{0}}$ and $\mathbf{R}_{\mathbf{0}}$ are determined in the $\mathrm{R}_{\mathrm{F}}$ reference frame as:

$\mathbf{S}_{\mathbf{0}\left(\mathrm{R}_{\mathrm{F}}\right)}=\left(\begin{array}{c}u_{S} \\ v_{S} \\ w_{S}\end{array}\right)=\left(\begin{array}{c}\cos a_{S} \cos h_{S} \\ \sin a_{S} \cos h_{S} \\ \sin h_{S}\end{array}\right) \quad$ and $\quad \mathbf{R}_{\mathbf{0}\left(\mathrm{R}_{\mathrm{F}}\right)}=\left(\begin{array}{c}u_{R} \\ v_{R} \\ w_{R}\end{array}\right)=\frac{1}{D}\left(\begin{array}{c}x_{R}-x_{H} \\ y_{R}-y_{H} \\ z_{R}-z_{H}\end{array}\right)$,

where $D=\sqrt{\left(x_{R}-x_{H}\right)^{2}+\left(y_{R}-y_{H}\right)^{2}+\left(z_{R}-z_{H}\right)^{2}}$ is the distance $\left\|\mathrm{OO}^{\prime}\right\|$ from the heliostat to the nominal target point.

3. The normal vector to the heliostat $\mathbf{N}_{\mathbf{0}}\left(u_{\mathrm{N}}, v_{\mathrm{N}}, w_{\mathrm{N}}\right)$ is then deduced from the SnellDescartes reflection law:

$\mathbf{N}_{\mathbf{0}}=\left(\mathbf{S}_{\mathbf{0}}+\mathbf{R}_{\mathbf{0}}\right) / \sqrt{2\left(1+\mathbf{S}_{\mathbf{0}} \mathbf{R}_{\mathbf{0}}\right)}$

4. The azimuth and elevation angles of vector $\mathbf{N}_{\mathbf{0}}$ are estimated as:

$a_{N}=\arctan v_{N} / u_{N}$ and $h_{N}=\arcsin w_{N}$.

5. The direction cosines of the Sun vector $\mathbf{S}_{\mathbf{0}}$ are transferred into the heliostat reference frame $\mathrm{R}_{\mathrm{H}}$. For an altazimuthal mount, it writes as the following matrix transform: 
$\mathbf{S}_{\mathbf{0}\left(\mathrm{R}_{\mathrm{H}}\right)}=\left(\begin{array}{c}u_{S\left(R_{H}\right)} \\ v_{S\left(R_{H}\right)} \\ w_{S\left(R_{H}\right)}\end{array}\right)=P_{\left(\mathrm{R}_{\mathrm{F}}\right) \rightarrow\left(R_{H}\right)} \mathbf{S}_{\mathbf{0}\left(\mathrm{R}_{\mathrm{F}}\right)}=\left[\begin{array}{ccc}\cos a_{N} \cos h_{N} & \sin a_{N} \cos h_{N} & \sin h_{N} \\ -\sin a_{N} & \cos a_{N} & 0 \\ -\cos a_{N} \sin h_{N} & -\sin a_{N} \sin h_{N} & \cos h_{N}\end{array}\right]\left(\begin{array}{c}\cos a_{S} \cos h_{S} \\ \sin a_{S} \cos h_{S} \\ \sin h_{S}\end{array}\right)$.

6. The angles $A$ and $H$ are finally derived as:

$A=\arctan v_{S\left(R_{H}\right)} / u_{S\left(R_{H}\right)}$ and $H=\arcsin w_{S\left(R_{H}\right)}$.
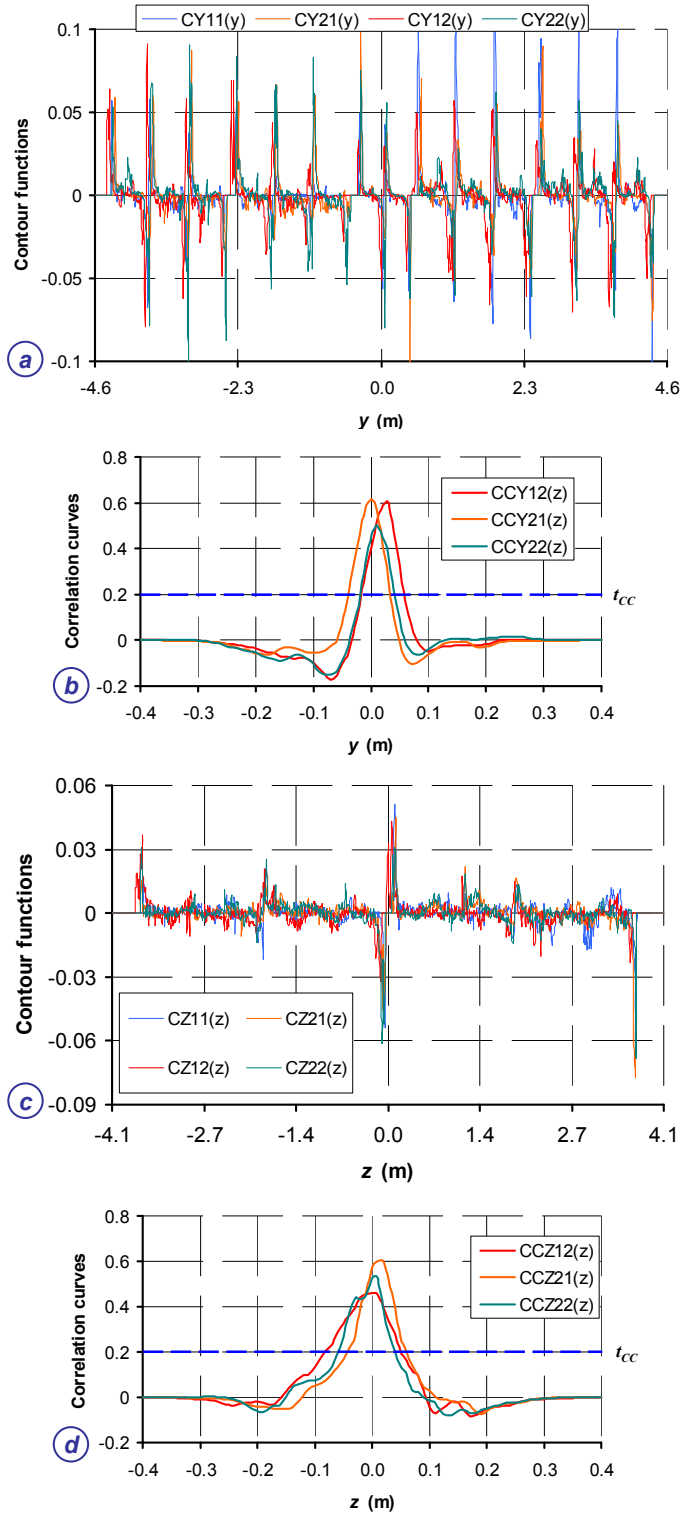

Figure B1: Illustrating the image registration procedure. (a) Contour functions along the Yaxis. (b) Cross-correlations along the Y-axis. (c) and (d) Same illustrations for registration along the Z-axis. 


\section{Appendix D. Reverse ray-tracing algorithm}

Detailed steps of the reverse ray-tracing algorithm are described below. Most of employed notations are illustrated in Figure 3 and some other ones are the same as in Appendix C.

1. Assuming a reference spherical heliostat of focal length $D$, the Cartesian coordinates of the vector $\mathbf{O P}$ are computed in the heliostat reference frame $\mathrm{R}_{\mathrm{H}}$ :

$\mathbf{O P}_{\left(R_{H}\right)}=\left(\begin{array}{c}x \\ y \\ z\end{array}\right)=\left(\begin{array}{c}r^{2} / 4 D+r^{4} / 64 D^{3} \\ y \\ z\end{array}\right)$

$$
\text { with } r^{2}=x^{2}+y^{2} \text {. }
$$

2. The coordinates of vector $\mathbf{O P}$ are transferred into the facility reference frame $\mathrm{R}_{\mathrm{F}}$ via the matrix relationship:

$$
\mathbf{O P}_{\left(R_{F}\right)}=P_{\left(R_{H}\right) \rightarrow\left(R_{F}\right)} \mathbf{O P}_{\left(R_{H}\right)}=\left(\begin{array}{c}
x_{P} \\
y_{P} \\
z_{P}
\end{array}\right)=\left[\begin{array}{ccc}
\cos a_{N} \cos h_{N} & -\sin a_{N} & -\cos a_{N} \sin h_{N} \\
\sin a_{N} \cos h_{N} & \cos a_{N} & -\sin a_{N} \sin h_{N} \\
\sin h_{N} & 0 & \cos h_{N}
\end{array}\right]\left(\begin{array}{l}
x \\
y \\
z
\end{array}\right),
$$

with $a_{N}$ and $h_{N}$ the azimuth and elevation angles of vector $\mathbf{N}_{\mathbf{0}}$ as defined in Appendix C.

3. The direction cosines $\left(u_{\mathrm{P}}, v_{\mathrm{P}}, w_{\mathrm{P}}\right)$ of a unitary vector $\mathbf{N}(\mathbf{P})$ perpendicular to the surface of the spherical heliostat at point $\mathrm{P}$ are computed in the heliostat reference frame (see Figure 3):

$$
\mathbf{N}(\mathbf{P})_{\left(R_{H}\right)}=\left(\begin{array}{l}
u_{P\left(R_{H}\right)} \\
v_{P\left(R_{H}\right)} \\
w_{P\left(R_{H}\right)}
\end{array}\right)=\frac{1}{d_{P}^{\prime}}\left(\begin{array}{c}
1 \\
\partial x(y, z) / \partial y-y\left(1+r^{2} / 8 D^{2}\right) / 2 D \\
\partial x(y, z) / \partial z-z\left(1+r^{2} / 8 D^{2}\right) / 2 D
\end{array}\right)
$$

where $\partial x(y, z) / \partial y$ and $\partial x(y, z) / \partial z$ are the optimization variables employed in section 2.2.8, and:

$$
d_{P}^{\prime}=\sqrt{1+\left(\partial x(y, z) / \partial y-y\left(1+r^{2} / 8 D^{2}\right) / 2 D\right)^{2}+\left(\partial x(y, z) / \partial z-z\left(1+r^{2} / 8 D^{2}\right) / 2 D\right)^{2}} .
$$

4. The direction cosines of vector $\mathbf{N}(\mathbf{P})$ are transferred into the facility reference frame using the same matrix as in relation $\mathrm{D} 2$ :

$\mathbf{N}(\mathbf{P})_{\left(R_{F}\right)}=P_{\left(R_{H}\right) \rightarrow\left(R_{F}\right)} \mathbf{N}(\mathbf{P})_{\left(R_{H}\right)}=\left(\begin{array}{l}u_{P\left(R_{F}\right)} \\ v_{P\left(R_{F}\right)} \\ \left.w_{P\left(R_{F}\right)}\right)\end{array}\right)=\left[\begin{array}{ccc}\cos a_{N} \cos h_{N} & -\sin a_{N} & -\cos a_{N} \sin h_{N} \\ \sin a_{N} \cos h_{N} & \cos a_{N} & -\sin a_{N} \sin h_{N} \\ \sin h_{N} & 0 & \cos h_{N}\end{array}\right]\left(\begin{array}{c}u_{P\left(R_{H}\right)} \\ v_{P\left(R_{H}\right)} \\ w_{P\left(R_{H}\right)}\end{array}\right)$.

The following steps are then carried out for each camera located at the four points $\mathrm{M}_{i j}$.

5. The coordinates of the vector $\mathbf{O}^{\prime} \mathbf{M}^{\prime}{ }_{i j}$ are transferred from the reference frame R" attached to the target plane (here tilted by 30 degs. around the $\mathrm{Y}_{\mathrm{F}}$-axis) to the facility reference frame $\mathrm{R}_{\mathrm{F}}$ :

$$
\mathbf{O}^{\prime} \mathbf{M}_{i j\left(R_{F}\right)}^{\prime \prime}=P_{\left(R^{\prime \prime}\right) \rightarrow\left(R_{F}\right)} \mathbf{O}^{\prime} \mathbf{M}_{i j\left(R^{\prime \prime}\right)}^{\prime \prime}=\left(\begin{array}{l}
x_{i j} \\
y_{i j} \\
z_{i j}
\end{array}\right)=\left[\begin{array}{ccc}
\cos \pi / 6 & 0 & -\sin \pi / 6 \\
0 & 1 & 0 \\
\sin \pi / 6 & 0 & \cos \pi / 6
\end{array}\right]\left(\begin{array}{c}
0 \\
y_{i j}^{\prime \prime} \\
z_{i j}^{\prime \prime}
\end{array}\right)
$$


6. In the $\mathrm{R}_{\mathrm{F}}$ reference frame, the unitary vector $\mathbf{R}_{i j}(\mathbf{P})$ directed along $\mathbf{P} \mathbf{M}_{i j}$ is computed as:

$\mathbf{R}_{i j}(\mathbf{P})_{\left(R_{F}\right)}=\frac{1}{D_{i j}}\left(\begin{array}{c}x_{R}+x_{i j}-x_{H}-x_{P} \\ y_{R}+y_{i j}-y_{H}-y_{P} \\ z_{R}+z_{i j}-z_{H}-z_{P}\end{array}\right)$,

where $\left(x_{\mathrm{P}}, y_{\mathrm{P}}, z_{\mathrm{P}}\right)$ and $\left(x_{i}, y_{i}, z_{i}\right)$ are obtained from relations $\mathrm{D} 2$ and $\mathrm{D} 5$ respectively, $\left(x_{\mathrm{R}}, y_{\mathrm{R}}, z_{\mathrm{R}}\right)$ and $\left(x_{\mathrm{H}}, y_{\mathrm{H}}, z_{\mathrm{H}}\right)$ are the coordinates of points $\mathrm{O}^{\prime}$ and $\mathrm{O}$ as defined in Appendix $\mathrm{C}$, and $D_{i j}=\sqrt{\left(x_{R}+x_{i j}-x_{H}-x_{P}\right)^{2}+\left(y_{R}+y_{i j}-y_{H}-y_{P}\right)^{2}+\left(z_{R}+z_{i j}-z_{H}-z_{P}\right)^{2}}$.

7. The Snell-Descartes reflection law is applied to vectors $\mathbf{N}(\mathbf{P})$ and $\mathbf{R}_{i j}(\mathbf{P})$ in order to calculate a unitary vector $\mathbf{S}_{i j}(\mathbf{P})$ retro-reflected towards the Sun disk at point $\mathrm{P}$ :

$\mathbf{S}_{i j}(\mathbf{P})_{\left(R_{F}\right)}=2\left(\mathbf{N}(\mathbf{P})_{\left(R_{F}\right)} \mathbf{R}_{i j}(\mathbf{P})_{\left(R_{F}\right)}\right) \mathbf{N}(\mathbf{P})_{\left(R_{F}\right)}-\mathbf{R}_{i j}(\mathbf{P})_{\left(R_{F}\right)}$

8. The direction cosines $\left(u_{i j}, v_{i j}, w_{i j}\right)$ of the vector $\mathbf{S}_{i j}(\mathbf{P})$ are transferred into the Sun reference frame $\mathrm{R}_{\mathrm{S}}$ via the matrix relation:

$\mathbf{S}_{i j}(\mathbf{P})_{\left(\mathrm{R}_{S}\right)}=\left(\begin{array}{c}u_{i j}\left(R_{S}\right) \\ v_{i j\left(R_{S}\right)} \\ w_{i j\left(R_{S}\right)}\end{array}\right)=P_{\left(\mathrm{R}_{F}\right) \rightarrow\left(R_{S}\right)} \mathbf{S}_{i j}(\mathbf{P})_{\left(\mathrm{R}_{F}\right)}=\left[\begin{array}{ccc}\cos a_{S} \cos h_{S} & \sin a_{S} \cos h_{S} & \sin h_{S} \\ -\sin a_{S} & \cos a_{S} & 0 \\ -\cos a_{S} \sin h_{S} & -\sin a_{S} \sin h_{S} & \cos h_{S}\end{array}\right]\left(\begin{array}{c}\left.u_{i j\left(R_{F}\right)}\right) \\ v_{i j\left(R_{F}\right)} \\ \left.w_{i j\left(R_{F}\right)}\right)\end{array}\right)$,

with $a_{\mathrm{S}}$ and $h_{\mathrm{S}}$ the azimuth and elevation angles of the Sun vector $\mathbf{S}_{\mathbf{0}}$ in the $\mathrm{R}_{\mathrm{F}}$ reference frame.

9. The $\varepsilon_{i j}(\mathrm{P})$ angle is finally computed as:

$\varepsilon_{i j}(P)=\sqrt{v_{i j\left(R_{S}\right)}^{2}+w_{i j}^{2}\left(R_{S}\right)}$ 